\title{
Origins Survey Spectrometer: revealing the hearts of distant galaxies and forming planetary systems with far-IR spectroscopy
}

\author{
Charles Matt Bradford, ${ }^{\mathrm{a}, *}$ Bruce Cameron, ${ }^{\mathrm{a}}$ Bradley Moore, ${ }^{\mathrm{a}}$ \\ Steve Hailey-Dunsheath, ${ }^{b}$ Edward Amatucci $\odot{ }^{\mathbf{c}}$ Damon Bradley, ${ }^{\mathrm{c}}$ \\ James Corsetti, ${ }^{c}$ David Leisawitz $\odot{ }^{c}$ Michael DiPirro, ${ }^{c}$ James Tuttle, ${ }^{c}$ \\ Ari Brown, ${ }^{\text {c }}$ Dick McBirney, ${ }^{\mathrm{c}}$ Alexandra Pope $\odot,{ }^{\mathrm{d}}$ Lee Armus, \\ Margaret Meixner, ${ }^{\mathrm{e}}$ and Klaus Pontoppidan $\odot^{\mathrm{e}}$ \\ a Jet Propulsion Laboratory, California Institute of Technology, Pasadena, \\ California, United States \\ ${ }^{\text {b}}$ California Institute of Technology, Pasadena, California, United States \\ ${ }^{c}$ NASA Goddard Space Flight Center, Greenbelt, Maryland, United States \\ ${ }^{\mathrm{d} U n i v e r s i t y ~ o f ~ M a s s a c h u s e t t s, ~ D e p a r t m e n t ~ o f ~ A s t r o n o m y, ~ A m h e r s t, ~ M a s s a c h u s e t t s, ~ U n i t e d ~ S t a t e s ~}$ \\ ${ }^{\text {e}}$ Space Telescope Science Institute, Baltimore, Maryland, United States
}

\begin{abstract}
The Origins Survey Spectrometer (OSS) is a multi-purpose far-IR spectrograph for Origins. Operating at the photon background limit, OSS covers the 25 - to 588- $\mu$ m wavelength range instantaneously at a resolving power $(R)$ of 300 using six logarithmically spaced grating modules. Each module couples at least 30 and up to 100 spatial beams simultaneously, enabling true [three-dimensional (3D)] spectral mapping. In addition, OSS provides two high-resolution modes. The first inserts a long-path Fourier-transform spectrometer (FTS) into a portion of the incoming light in advance of the grating backends, enabling $R$ up to $43,000 \times[\lambda / 112 \mu \mathrm{m}]$, while preserving the grating-based sensitivity for line detection. The second incorporates a scanning etalon in series with the FTS to provide $R$ up to 300,000 for the 100 -to $200-\mu \mathrm{m}$ range. (C) The Authors. Published by SPIE under a Creative Commons Attribution 4.0 Unported License. Distribution or reproduction of this work in whole or in part requires full attribution of the original publication, including its DOI. [DOI: 10.1117/1.JATIS.7.1.011017]
\end{abstract}

Keywords: Origins; far-infrared spectroscopy; survey spectroscopy; direct-detector.

Paper 20083SS received Jun. 15, 2020; accepted for publication Jan. 27, 2021; published online Mar. 11, 2021.

\section{Introduction}

The Origins Space Telescope (Origins) traces our cosmic history, from the formation of the first galaxies and the rise of metals to the development of habitable worlds and present-day life. Origins does this through exquisite sensitivity to infrared radiation from ions, atoms, molecules, dust, water vapor and ice, and observations of extrasolar planetary atmospheres, protoplanetary disks, and large-area extragalactic fields. Origins operates in the wavelength range 2.8 to $588 \mu \mathrm{m}$ and is 1000 times more sensitive than its predecessors due to its large cold $(4.5 \mathrm{~K})$ telescope and advanced instruments.

\subsection{Scientific Motivation for Far-IR Spectroscopy}

A number of high-priority Origins scientific investigations require sensitive, wide-bandwidth spectroscopy covering the full the far-IR. The extragalactic science cases, in particular, center around charting the cosmic history of the contents and processes within galaxies from the first billion years to the present day using rest-frame mid- and far-IR spectral tracers. The various mid-IR and far-IR features originate from a wide range of interstellar medium phases: ionized, neutral atomic, and molecular material, and in aggregate they constrain heavy-element contents,

*Address all correspondence to Charles Matt Bradford, bradford@caltech.edu 
local UV radiation field conditions, which reveal stellar properties and physical conditions (temperature and density). ${ }^{1-3}$ Crucially, the features in this waveband are largely immune to dust obscuration, providing an unbiased census of the interplay between stars, black holes, and the interstellar material in any given observation.

Of particular interest for Origins, and driving the sensitivity, is the epoch of reionization and the dawn of galaxies' first billion years. As the Universe was enriched from primordial hydrogen and helium to a medium that contains heavy elements and dust grains, the key cooling pathways shifted from the quadrupole pure rotational $\mathrm{H}_{2}$ lines $(28,17,12,9.7,8.0,6.9, \ldots, \mu \mathrm{m})($ e.g., Refs. 4 and 5) to a combination of atomic fine-structure transitions and dust thermal emission. Origins will probe both sides of this transition out to $z \sim 8$ to 10 using $\mathrm{H}_{2}$ lines, fine-structure lines, and polycyclic aromatic hydrocarbon (PAH) features at 6.2 to $17 \mu \mathrm{m}$, which have $15 \times$ more power than the brightest atomic cooling lines. ${ }^{6}$ Both the $\mathrm{H}_{2}$ lines and most of the PAH features are redshifted out of the James Webb Space Telescope (JWST) band, but not into the atacama large millimeter array (ALMA) windows in the $z \sim 5$ to 10 era. Origins with the Origins Survey Spectrometer (OSS) spectrograph will detect these powerful bands at early epochs (Fig. 7).

Measuring these features does not require high spectral resolution, as integrated line fluxes are all that is required. However, accessing the full-time history from the first billion years to the present day does require both exquisite sensitivity (because of $1 / d_{\text {Luminosity }}^{2}$ dimming) and coverage across the full far-IR to capture the wide intrinsic wavelength distribution of spectral features-approximately uniformly in $\log \lambda$ and considering the large range of redshifts.

In addition to pointed observations of individual sources and fields identified a priori, similar to what is done with ALMA and will be done with JWST, the OSS on Origins is designed to be a powerful wide-field spectral mapper. In its blind survey mode, millions of galaxies will be identified with redshifts and physical parameters immediately encoded. ${ }^{10}$ These requirements set the basic architecture for the OSS: a suite of $\mathcal{R}=300$ wideband long-slit grating spectrometers with detectors operating at or near the fundamental limits.

The resulting excellent sensitivity to spectral line surface brightness (velocity-integrated, spatially unresolved line emission with units of $\mathrm{Wm}^{-2} \mathrm{sr}^{-1}$, e.g., from diffuse gas or unresolved faint galaxies) also enables three-dimensional (3D) intensity mapping of residual line emission fluctuations after individual sources are extracted. The resulting intensity maps can yield the total cosmic luminosity density - including all sources even those too faint to detect individually - in the key far-IR lines, charting aggregate properties of galaxies through time. ${ }^{11-17}$

For pointed observations, the high-resolution mode is also available (see below). This offers a direct measure of stellar and black-hole feedback through outflow signatures in $\mathrm{OH}$ and other transitions, potentially a key ingredient in the decline of the cosmic star formation rate density over the last 8 billion years. ${ }^{18}$

Another driving case for the OSS is understanding the formation of planetary systems. This requires tracking the evolution of gas mass and composition in protoplanetary disks from the early (protostellar) phase to the late (debris-disk) stages where most of the gas has been dispersed or absorbed into giant planets. For early stage, gas-rich disks, the fundamental of hydrogen deuteride (HD) at $112 \mu \mathrm{m}$ is an excellent probe of total mass. It is an optically thin chemical analog of $\mathrm{H}_{2}$ and has only a modest temperature dependence. The challenge for studying protoplanetary disks is not so much the raw spectral line sensitivity-even the relatively weak HD integrated line flux is typically high by comparison with lines in distant galaxies-but extracting line fluxes accurately in the face of a bright dust continuum. HD, in particular, can be easily washed out by the bright disk continuum so it requires a high resolving power ( $R \sim$ several thousands) for reliable measurement. Gas mass evolution is the first step, but with Origins we will also study the conditions for Earth-like habitability by tracking water in disks with low-lying far-IR transitions that probe the bulk of the gas including that in the snow line. Distinguishing material interior to the snow line from material further out requires line-width measurements with a resolving power of 25,000 at wavelengths as long as $179 \mu \mathrm{m}$, a key ground-state water transition. These higher-resolving-power requirements are satisfied with the addition of a Fourier-transform spectrometer (FTS) which operates in tandem with the grating spectrometer suite. Finally, in order to study the structure of disks through Doppler tomography, the OSS includes a veryhigh-resolution mode using an etalon in concert with the FTS to provide $\delta v \sim 1$ to $2 \mathrm{~km} / \mathrm{s}$ for HD $112 \mu \mathrm{m}$ and $\mathrm{H}_{2} \mathrm{O} 179 \mu \mathrm{m}$. 


\section{OSS Functional Approach}

Figure 1 shows the OSS functional approach. At the heart of the instrument are six slit-fed wideband grating modules, which combine to span the full 25 to $588 \mu \mathrm{m}$ range. These grating modules have no moving parts and will be configured so that the six slits are co-aligned on the sky. Although the beam sizes (setting the slit widths) and the slit lengths are not the same from band to band, the co-alignment enables point sources incident in the band 1 slit to be simultaneously measured in the longer-wavelength bands (see Fig. 5 in Leisawitz et al., this volume shows the Origins focal plane configuration). The wide-band echelle gratings, particularly the long-wavelength ones, which require compact designs, typically only have high blaze efficiency in one linear polarization, so the gratings are used in single polarization. Light from the telescope first encounters a polarizing grid; one linear polarization is passed into one arm while one is reflected into a separate arm. One arm then feeds bands 1, 3, and 5, whereas the other arm feeds bands 2, 4, and 6. Staggering odd- and even-numbered bands allows high-efficiency dichroic filters to separate the bands and allows the bands to overlap slightly in the two polarizations. Since the high-efficiency polarization is that with the electric field perpendicular to the slit direction, either the even or odd bands will require polarization rotation at the entrance to the slit. This can be accomplished with broadband half-wave plates for which there is a rich heritage in the millimeter band ${ }^{19-22}$ using multiple crystal plates and metal mesh; techniques are amenable to operation at shorter wavelengths. More advanced metamaterial approaches have also demonstrated good broadband performance. ${ }^{23-25}$

The grating suite is used in point-and-chop mode for deep observations of individual objects. Here the observatory field steering mirror (FSM) modulates the image of a point source between two positions on the slit. The grating modules also operate as a mapping instrument, in a manner similar to Herschel/SPIRE, ${ }^{26}$ where detectors sample the sky as the slit is rastered around. For OSS on Origins, this is accomplished either using the FSM for small fields [on order the telescope field-of-view (FOV)] or for large fields by scanning the telescope itself as was done for Herschel/SPIRE. Table 1 shows the high-level instrument parameters, sensitivity, and mapping speed estimates for the base grating system of OSS.

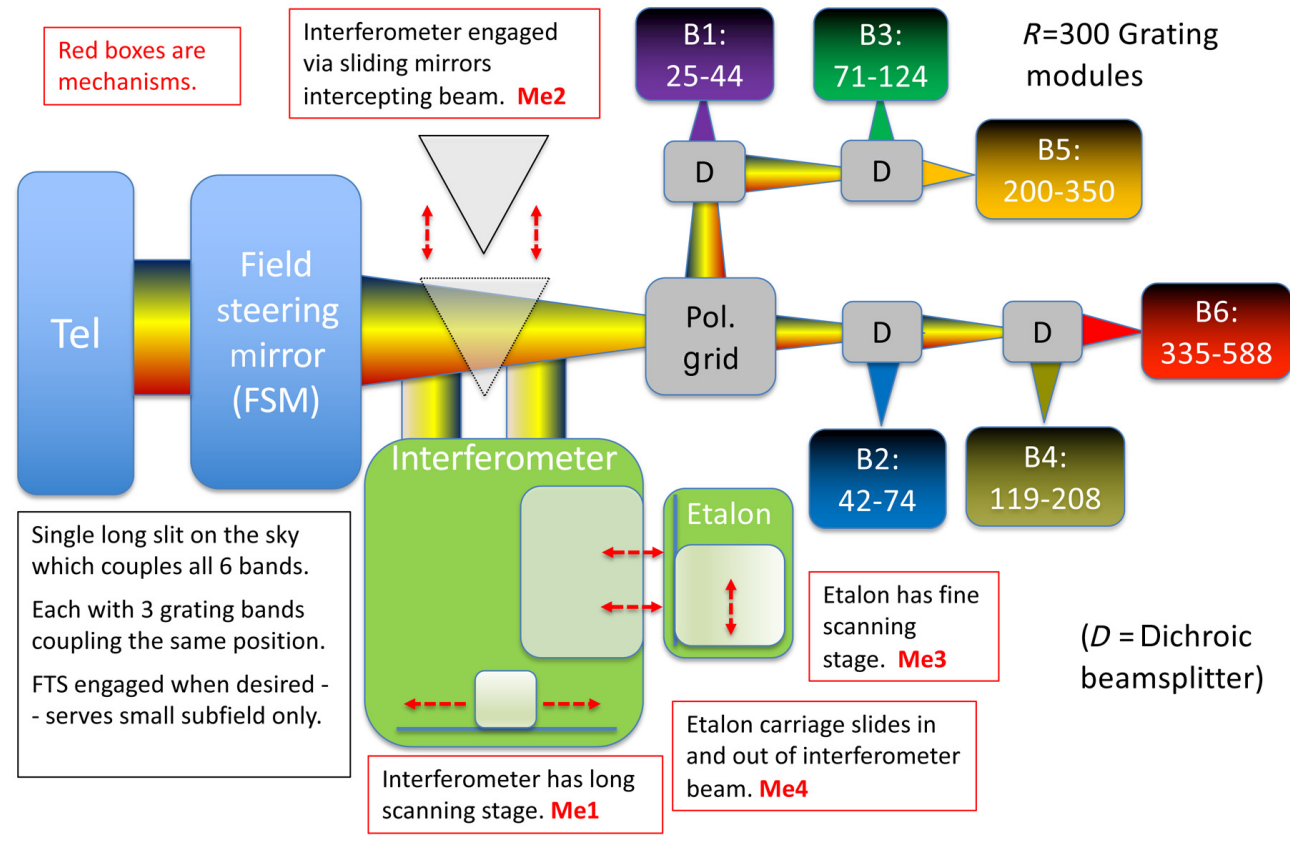

Fig. 1 The OSS functional block diagram shows the six grating modules with their integrated detector arrays, polarizing grid, and dichroic filters (all fixed, with no mechanisms). The beam steering mirror (also known as the FSM) is part of the telescope. It provides chopping and enables small maps using the grating slits. For high-spectral-resolution measurements over a small subfield, an interferometer is inserted into the train with a sliding mechanism with two mirrors on it. The interferometer is a Martin-Puplett polarizing Fourier-transform system with an $8 \times$ path multiplier. The baseline design includes an etalon (Fabry-Perot interferometer) that can be inserted into the interferometer beam to enable very high resolution for Doppler tomography experiments. 
Bradford et al.: Origins Survey Spectrometer: revealing the hearts of distant galaxies...

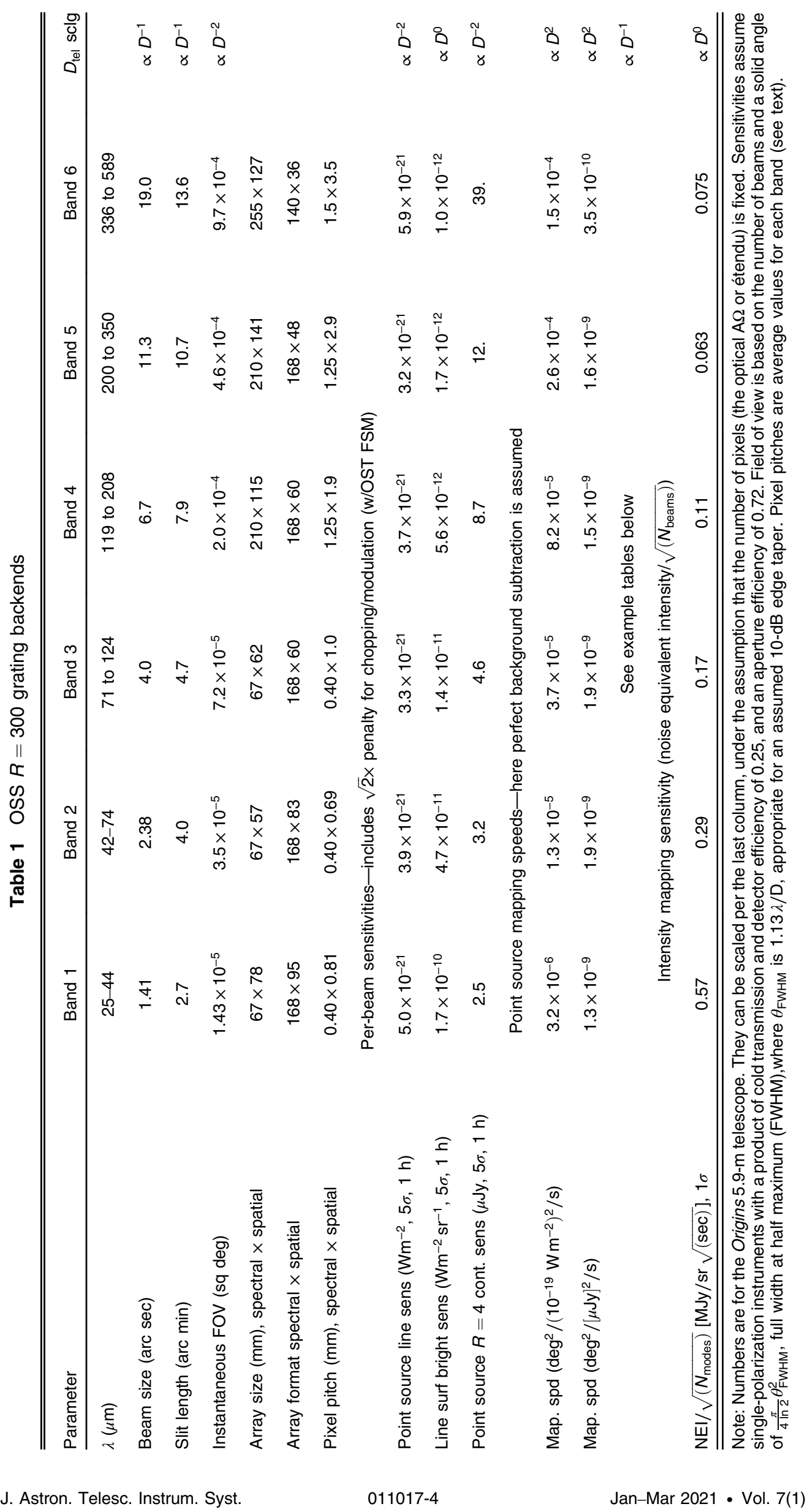


To provide higher spectral resolving power for individual sources, the interferometer is engaged for a small field common to all bands. A sliding carriage moves two mirrors into the beam: the first diverts the beam to the interferometer optics and the second reinserts it into the beam so it is detected by the grating backends. The interferometer is a Fourier-transform system designed to provide $7.5 \mathrm{~km} / \mathrm{s}$ resolution at the $112-\mu \mathrm{m}$ HD rotational transition to match outerdisk line widths. Using the gratings in tandem with the FTS preserves the good underlying line sensitivity of the small per-detector bandwidth $(R=300)$. Additionally, the interferometer includes an insertable etalon (Fabry-Perot interferometer) that can further improve the resolving power, enabling velocity-resolved measurements of HD and water in protoplanetary disks. This system targets $1 \mathrm{~km} / \mathrm{s}$ at $112 \mu \mathrm{m}$. The etalon cavity operates in high order and is scanned to produce a spectrum. These modes are described in detail in Sec. 4.3.

\section{Optical Design}

\subsection{Grating Module Designs}

Each grating module forms a light-tight enclosure that includes the detector array and for which the only opening is a slit. Requirements that met by the optical design include as follows.

- Slit length on sky. At least 100 diffraction-limited beams for bands 1 to 5, and 75 beams for band 6 (this provides margin — not all of this optical field is used in the baseline design because of readout limitations).

- Intrinsic spectrometer resolving power $\lambda / \delta \lambda$ of at least 300 over a 1:1.75 bandwidth.

- Strehl $>80 \%$ (goal $>90 \%$ ) imaging to a focal plane that images a $1.15 \lambda / D$ spatial $\times R=$ 300 spectral element (band-averaged) onto an area no smaller than $0.25 \mathrm{~mm}^{2}$ (to enable multiplexed detector readout).

The optical surfaces in the spectrometers are numerically optimized with many ( 20$)$ degrees of freedom, essentially approaching free-form surfaces, which is necessary to accommodate the large range of spatial and spectral fields. This approach is fully consistent with the machined aluminum construction. No constraints are placed on distortion in the focal plane, or "smile," which is the departure from a straight line of the spectrum from a given spatial position. This is because imaging a slit to a slight arc on a top-dimensional (2D) rectangular pixel array simply results in a slight wavelength shift of the pixels as one moves along the slit direction in the focal plane, an issue easily handled in software and potentially providing a spectral sampling benefit. Alternatively, if desired, a focal plane could be built with a custom arrangement of pixels in curved rows to match a curved slit image, since each focal plane is already fully custom. In any case, the slit shape at the entrance to the spectrometer (and thus on the sky) need not be curved. The size and mass of the short-wavelength gratings are driven primarily by the pixel pitch and thus the array size, whereas the long-wavelength modules are driven by the size of the grating and associated pupil. Figure 2 shows the shortest and longest of the grating module designs. The strawman detector array formats are tabulated for each band in Table 1, under the assumption of $1.13 f \lambda$ sampling spatially by $R=300$ sampling spectrally. As is typical of wideband grating systems that use a large incidence angle, the systems are highly anamorphic, with spatial focal ratio $(f / \#)$ much faster $(\sim 2 \times)$ than the spectral focal ratio $(f / \#)$. Pixels are, therefore, rectangular, elongated in the spatial direction. Additionally, the physical size of an $R=300$ bin changes across the band, so each focal plane will have at least two spectral pitches across the full band to preserve a given fractional bandwidth per pixel.

\subsection{Interferometer Design}

The design of the interferometer (Fig. 3) is driven by the need to incorporate high spectral resolution at the long OSS wavelengths while maintaining the excellent sensitivity and multi-band capability of the base grating suite. The approach is to intercept the full-band light from a small field and process it with a FTS before sending it to the grating modules for detection. This ensures that the basic sensitivity to spectral lines provided by the $R=\nu / \delta \nu=\lambda / \delta \lambda=300$ 


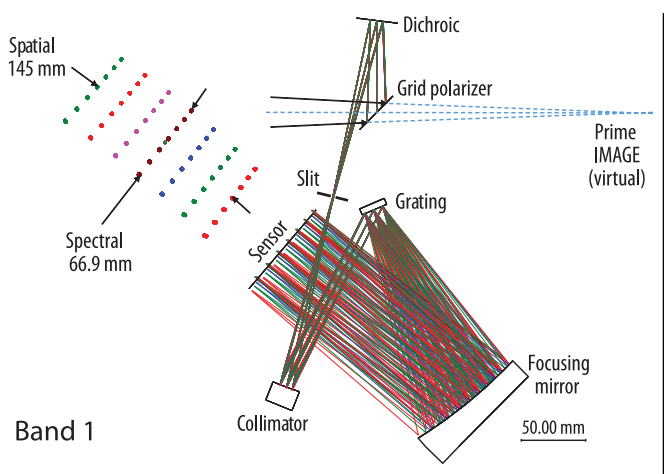

(a)

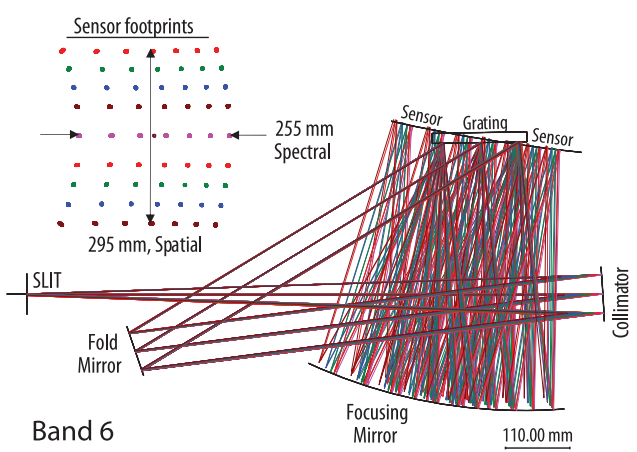

(b)

Fig. 2 Grating module designs for (a) band 1 and (b) band 6, the shortest- and longest-wavelength bands in OSS. In both cases, the $f / 14.4$ telescope focus is shown, positioned at the input slit for band 6 , and the conjugate to it for band 1 . The grating optical designs and focal-plane dimensions shown here represent larger focal planes than are currently baselined (pixel count is limited by readout), and the optics accommodate these, thereby allowing for an upscope. Band 1 in particular can accommodate a large slit up to 250 beams. The band 6 focal plane uses the four spatial fields above and below the center, which is obscured by the grating.
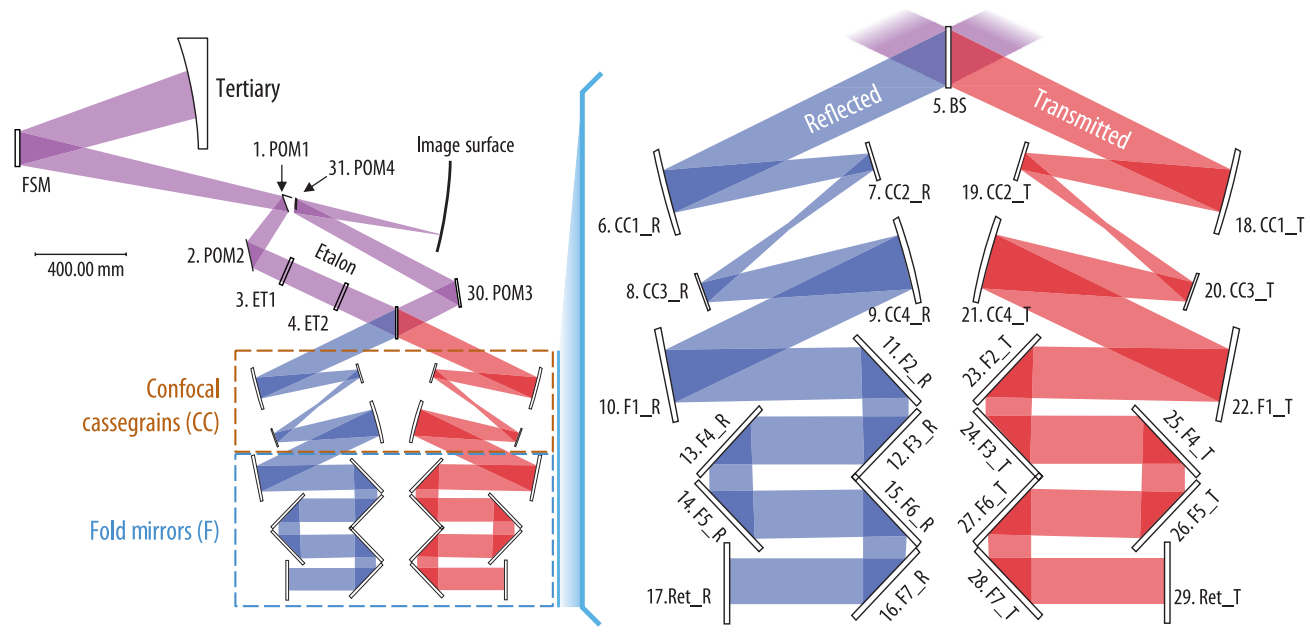

Fig. 3 Optical layout for the OSS interferometer. Light is intercepted in the converging beam from the telescope and is collimated with a diameter of $8 \mathrm{~cm}$. After processing by the interferometer, the light is reinserted into the original light path from the telescope to the grating modules. The interferometer is engaged with a sliding stage containing POM1 and POM4. It only accesses a small portion of the grating slits as it is designed primarily for single-source or small-field measurements. On the right, the mirrors are numbered. The single moving stage carries mirrors 11, 12, 15, 16, 23, 24,27 , and 28. A detail not depicted in this figure is the retroreflectors 17 and 29; they are roof-top mirrors oriented at $45 \mathrm{deg}$ with respect to the interferometer plane to provide the polarization rotation (see Ref. 28).

detector bandwidth is preserved. The system can be considered as an extreme implementation of a band-limited FTS with many output bands.

The baseline design has a $30-\mathrm{cm}$ physical throw providing $2.4 \mathrm{~m}$ of optical path difference (OPD) between the arms of the interferometer. This provides a wavelength-dependent maximum resolving power of $43,000 \times[112 \mu \mathrm{m} / \lambda]$, a good match to expected linewidths of the HD $112 \mu \mathrm{m}$ transition in protoplanetary disks (see Origins study report, Sec. 1.2.2.5). Shorter FTS scans can be employed; this reduces $R$, but the $1 / \lambda$ dependence is always present since the single FTS scan is common to all six bands. The collimated beam diameter is $8 \mathrm{~cm}$, which ensures divergence (diffraction) is not a concern for the FTS operation, even at long wavelengths. The long-extra 
optical path between the pickoff/reinsertion position and the interferometer retroreflectors necessitates pupil reimaging to keep the system compact. This is provided by two sets of confocal off-axis Cassegrain telescopes that image the telescope pupil to the back of the interferometer. The FTS architecture is a Martin-Puplett polarizing FTS (Refs. 27; 28), which is optimal for this application. Relative to other approaches to Fourier-transform spectroscopy, this system uses a single-input port and single-output port. It requires a static polarizing grid at the input prior to the beam splitter (e.g., at or near POM2), and a linearly polarized detector, but both output polarizations can be used with independent detectors, exactly as is baselined for the OSS gratings. In each of these output polarizations, the system produces an interferogram with no lost light, unlike a Michelson architecture, which shares power between two ports.

In addition to the FTS, a second, higher-resolution capability enables line-profile Doppler tomography measurements of $\mathrm{HD}$ and $\mathrm{H}_{2} \mathrm{O}$ lines around the $112-\mu \mathrm{m}$ band. (Operation at other wavebands may be possible, a subject for phase-A study.) This ultra-high-resolution mode is provided with an etalon (Fabry-Perot interferometer) consisting of a 26-cm long cavity formed by two partially reflecting mirrors that can be inserted into the beam before the FTS beam splitter. With a design finesse of 70, the etalon provides a resolving power of $325,000(0.9 \mathrm{~km} / \mathrm{s})$ at $112 \mu \mathrm{m}$, or $200,000(1.5 \mathrm{~km} / \mathrm{s})$ at $179 \mu \mathrm{m}$. The etalon uses the same $8-\mathrm{cm}$ collimated beam provided for the FTS; however, to allow for beam walk in the etalon without substantial signal loss, the mirrors must be $14 \mathrm{~cm}$ in diameter. The mirrors in the FTS are similarly oversized. Once in the optical train, the cavity spacing can be changed by $\sim 1 \mathrm{~mm}$, providing a wavelength scan of a few orders, which is ample to provide complete coverage. The etalon mirrors will either be stretched metal mesh with high heritage (Sec. 10), and recent demonstrations of $\mathcal{R}=100,000,{ }^{29}$ or more modern patterned silicon, ${ }^{30}$ which could improve performance for wider band coverage.

\subsection{Full Optical Configuration}

Since all six grating modules must be fed through their slits at the telescope focus, and these slits overlap, packaging is an important consideration, and the solution has been obtained through careful iteration with the observatory mechanical and optical designs. Figure 4 shows views of

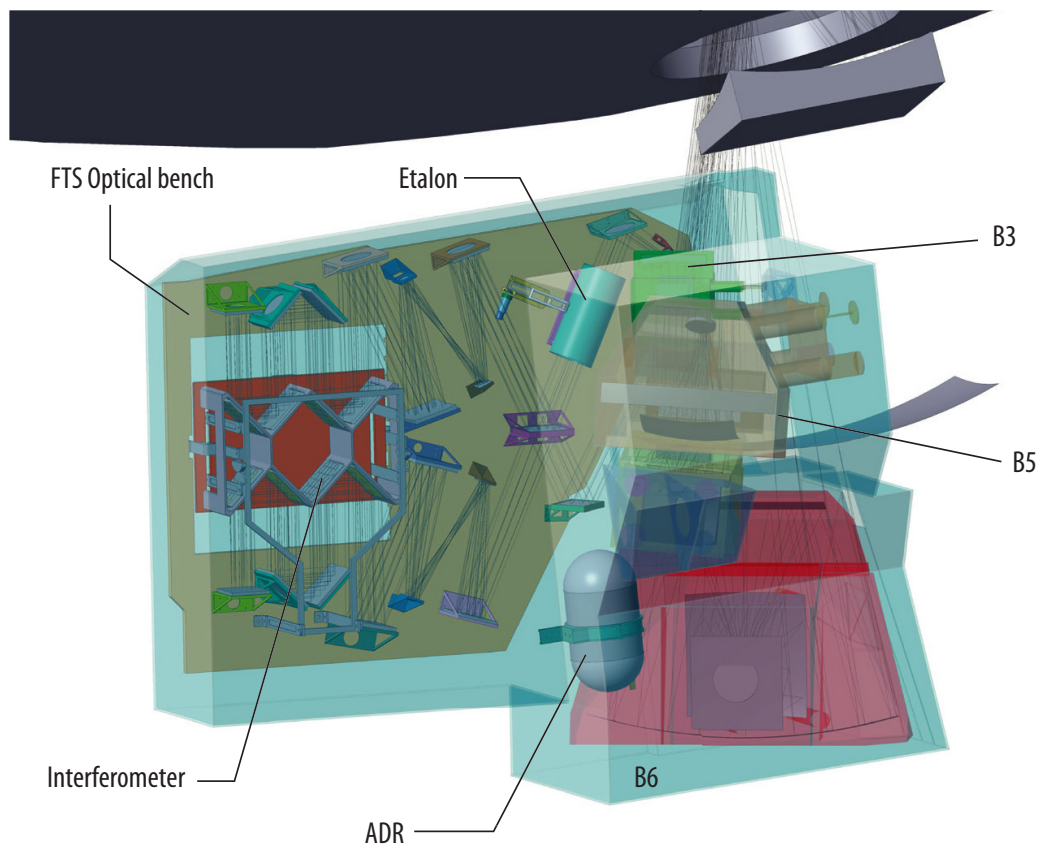

Fig. 4 The OSS optical and mechanical design has at its core, the six grating models (right). Light from the telescope comes in from the top (blue rays) and is split into the six bands via polarizing grids and dichroics. The FTS spectrometer (back left) picks off light near the entrance of the instrument, passing it through the interferometer and then back through the grating modules. For the highest spectral resolution, an etalon (turquoise tube) is inserted in the light departing from the FTS. 
Bradford et al.: Origins Survey Spectrometer: revealing the hearts of distant galaxies...

Table 2 OSS observing mode summary

\begin{tabular}{lccccc}
\hline \hline Mode & Band & $R$ & FoV & Modulation & \multicolumn{1}{c}{ Notes } \\
\hline Low-resolution pointed & Full & 300 & Full slits & FSM chop along slit & $\begin{array}{l}\text { Best pointed-object } \\
\text { sensitivity }\end{array}$ \\
Low-resolution mapping & Full & 300 & Full slits & FSM or tel. scan & $\begin{array}{l}\text { Maximum spectral } \\
\text { mapping speed }\end{array}$ \\
High-resolution pointed & Full & $\begin{array}{l}43,000 \times \\
112 \mu \mathrm{m} / \lambda\end{array}$ & $\begin{array}{l}20 \text {-arc } \\
\text { min slit }\end{array}$ & FTS scan & $\begin{array}{l}R \text { is tunable via } \\
\text { scan length }\end{array}$ \\
Ultra-high-resolution & $\begin{array}{l}100 \text { to } \\
200 \mu \mathrm{m}\end{array}$ & $\begin{array}{l}300,000 \times \\
112 \mu \mathrm{m} / \lambda\end{array}$ & 1 beam & FTS scan & $\begin{array}{l}\text { 200 seconds for } \\
\text { each position }\end{array}$ \\
Bright source & $\begin{array}{l}100 \mathrm{to} \\
200 \mu \mathrm{m}\end{array}$ & 300 & $\begin{array}{l}20 \text {-arc } \\
\text { min slit }\end{array}$ & FSM chop & Etalon as flux reducer \\
\hline \hline
\end{tabular}

the full configuration with all grating bands, the interferometer, and etalon, as well as the field of view of the OSS in the Origins focal plane.

\section{Observing Modes and Detector Requirements}

Table 2 summarizes the OSS observing modes. The base $R=300$ long-slit spectroscopy mode can be used for pointed observations of targets of interest and for mapping.

\subsection{Pointed Low-Resolution Spectroscopy}

OSS is very efficient for pointed observations since the slits from all six bands overlap so that a complete spectrum is obtained over the full wavelength range simultaneously. For this mode, the Origins field steering mirror (FSM) will be used to chop a source back and forth with a selectable throw. For point sources, a distance of three long-wavelength beams or 1 arc min ensures that the flux in from an unresolved target source is negligible in the off-source position. The chop throw could extend up to the length of the short-wavelength (band 1) slit-2.7 arc min to ensure full efficiency with the source always coupled through the slit. Chop frequencies in this mode are expected to be as low as $0.5 \mathrm{~Hz}$, as the detectors will have good stability to this level. The Origins FSM is capable of faster chopping (up to $10 \mathrm{~Hz}$ ) if needed to overcome other sources of variability.

\subsection{Mapping Modes}

The OSS slits can also be rastered around the sky to generate maps. In this mode, the scan rate must not be so fast that the short-wavelength beams smear when sampled with the finite bandwidth of a detector. These modes together with the observatory scan speed, therefore, set a requirement on the detector time constant $\tau$. As a compromise between agility and the need to have a reasonable attitude control system, Origins selected $60 \mathrm{arcsec} / \mathrm{s}$ as the maximum observatory scan speed. Operating at this maximum speed then requires $\tau$ at the short wavelength $(25 \mu \mathrm{m})$ of at most $5 \mathrm{~ms}$, which is comfortably within the Origins $3 \mathrm{~ms}$ requirement. This maximum speed allows the low-frequency $f_{\text {knee }}$ to be as high as $17 \mathrm{mHz}$ for recovering the large-scale structure in the intensity mapping experiments. The 3-ms requirement has been comfortably met with all of the detector options under consideration.

\subsection{High-Resolution Mode}

With using the FTS, the modulation is naturally provided by the OPD change, so chopping is not required. A given rate of change of the OPD scan $v_{\mathrm{OPD}}$ connects the narrow spectral bandwidth of each grating channel to a correspondingly narrow audio-band signal via

$$
f_{\text {audio }}=v_{\text {OPD }} / \lambda,
$$


so, each $R=300$ detector needs only carry a signal in a narrow $(R=300)$ band about the central fringe rate. This provides some natural immunity against systematics and instabilities. In particular, absolute stability is not required over the full scan time. However, to limit the signal loss to $70 \%$ at the shortest wavelength, the fringe rate should be less than $(2 \pi \tau)^{-1}$, thus the limiting scan speed is $v_{\text {OPD,max }}=\lambda_{\min } /(2 \pi \tau)$, which is $1.3 \mathrm{~mm} / \mathrm{s}$ using the 3 -ms time constant. This scan rate results in a fringe rate at $580 \mu \mathrm{m}$ of $2.2 \mathrm{~Hz}$, well above the knee frequency. A scan of the full 2.4-m OPD then requires $30 \mathrm{~min}$, if the shortest wavelengths are to be recovered. If the recovery of the high-resolution information at the shortest wavelengths is not desired, then the scan could be quicker (e.g., $7 \mathrm{~min}$ for a scan recovering wavelengths down to $112 \mu \mathrm{m}$ ). Beam divergence in the interferometer, combined with practical size limitations of the optical design, limits the field of view through the interferometer. The high-resolution mode will couple a subslit of $\sim 20 \mathrm{arc} \mathrm{sec}$ through the interferometer and will be capable of providing the full resolving power over this small slit. For the longest wavelengths, this corresponds to a single beam, but for all shorter-wavelength bands, multiple spatial pixels will be available for background subtraction. At the longest wavelengths, the background is dominated by the stable cosmic microwave background, and with the large beam, the variability induced by pointing drifts while looking at a point source will be small, since the pointing spec for Origins (50 mas for both rms 1 to $10 \mathrm{~h}$ drift and $>1 \mathrm{~Hz}$ jitter) is driven by the mid-IR transit work and is much smaller than the far-IR beam (1.4 to $19 \operatorname{arc~sec})$.

\subsection{Very-High-Resolution Mode}

When using the etalon in the nominal design with cavity finesse $=70$ and cavity spacing of $26 \mathrm{~cm}$, the order number $m_{\mathrm{FP}}$ will be several thousand (e.g., $m_{\mathrm{FP}}=4600$ at $\mathrm{HD} 112 \mu \mathrm{m}$ ). Multiple orders will thus be coupled simultaneously to a single $R=300$ grating channel (e.g., 4600/300 15 at $112 \mu \mathrm{m}$ ), and an FTS scan will be required to resolve these orders from one another. To fully ensure spectral purity, each step in a high-resolution etalon spectrum will, therefore, require an order-sorting FTS scan. This scan, however, need only be long enough to clearly resolve the orders from one another, that is it requires $R=2 \times m_{\mathrm{FP}}$ or 10,000 at $\mathrm{HD}$ $112 \mu \mathrm{m}$. This is about $1 / 4$ of the total FTS range. Additionally, the etalon is not required to operate at wavelengths shorter than $100 \mu \mathrm{m}$, so the fringe rate constraint described above only applies to $100 \mu \mathrm{m}$. Thus, $v_{\mathrm{OPD} \text {,max }}$ for the order sorting operation is $5.3 \mathrm{~mm} / \mathrm{s}$, and the ordersorting scan requires $\sim 196 \mathrm{~s}$. A 20-position etalon scan will thus require $1.1 \mathrm{~h}$, and a 140-position etalon scan covering a full free spectral range (the space between orders) to produce a Nyquistsampled complete spectrum requires 140 positions, or nearly $8 \mathrm{~h}$. A subject for phase-A study is the potential to reduce this time with a custom FTS scan, which uses many fewer (e.g., 60) discrete positions sampled sparsely across the scan instead of a continuous or fully-sampled scan. This is expected work because the FTS scan only needs to discriminate among the few orders within a single $\mathcal{R}=300$ grating channel, so aliasing signals from much higher or lower frequencies, as is produced when large steps are taken in the FTS scan, would not couple to the detector.

\section{Detector System}

Unlike the near-IR and mid-IR detector arrays, far-IR to millimeter-wave detectors suitable for a mission like Origins do not have a commercial or military application, so developments are led by science-driven teams, with NASA playing a leading role. Additionally, the cold space telescope presents a uniquely low background, and the OSS per-pixel sensitivity target is two orders of magnitude below any devices yet fielded (Fig. 5). A less fundamental but equally important requirement is the format - the OSS has a total of 60,000 pixels in the six arrays and room in the optical/mechanical design for $2 \times$ more if the multiplexing system could allow it within the thermal constraints. Finally, we note the requirement on dynamic range: the OSS must be capable of spectroscopy of comets with flux densities up to $5 \mathrm{Jy}$ in the 100- to $150-\mu \mathrm{m}$ range. With the OSS telescope, this translates to a factor of 1500 above the zodiacal light background loading $\left(\sim 6 \times 10^{-19} \mathrm{~W}\right.$ per pixel). The OSS requirements could be met with multiple detector approaches, and our technology development plan is targeting three related technologies, each described in more detail in a separate article in this volume. In order of date of invention, they are as follows. 

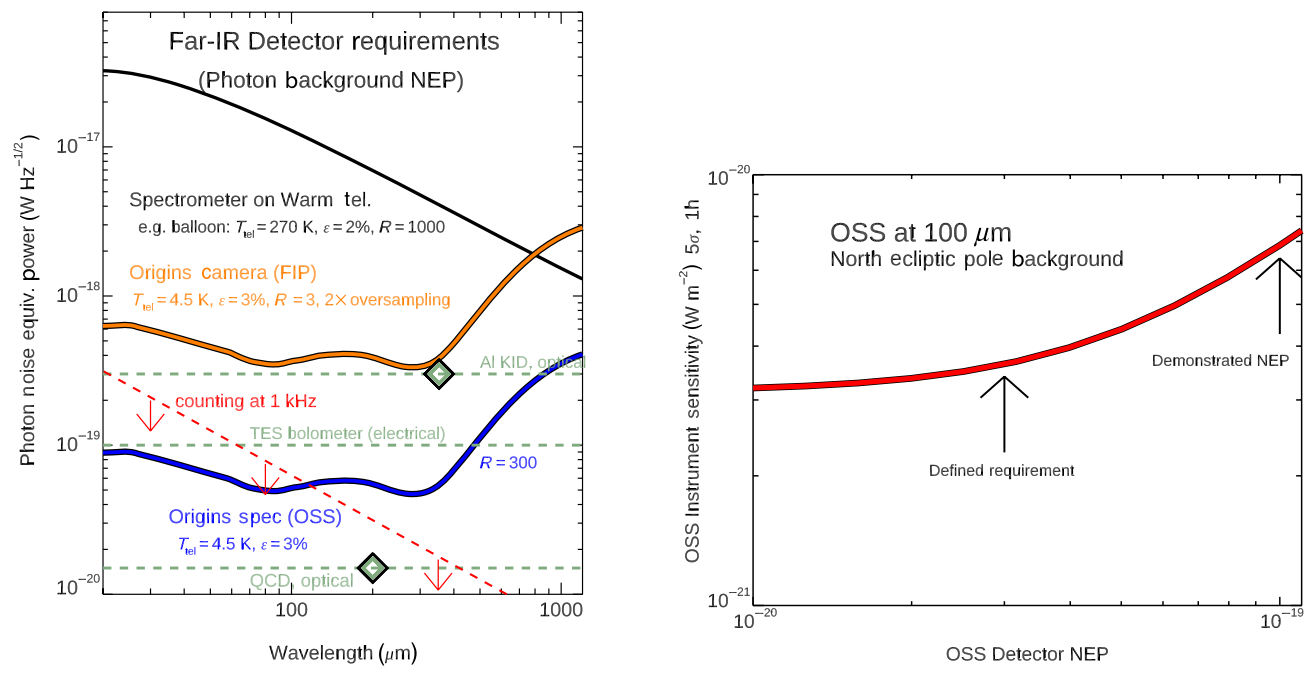

Fig. 5 Sensitivity [i.e., noise equivalent power (NEP)] requirements for far-IR instrumentation. Curves show the photon shot noise-improving detectors beyond this produces diminishing gains. The most demanding application is the Origins spectrometer (OSS), for which the photon NEP is as low as $3 \times 10^{-20} \mathrm{~W} \mathrm{~Hz}^{-1 / 2}$. Horizontal lines show published measurements (see text). A device that is both sensitive and fast becomes a photon counter, which offers the potential to both enable shot noise measurements at very high resolving power (e.g., with the OSS etalon) and overcome potential detector and readout system issues such as temperature and gain instability. Right shows the graceful degradation of the base OSS sensitivity as NEP increases by a factor of $\sim 3$ from the target.

Superconducting transition-edge-sensed (TES) bolometers. ${ }^{31}$ For OSS, the key virtue of the TES system is the significant heritage. TES arrays have been used in a variety of ground ${ }^{32-35}$ and suborbital platforms ${ }^{36}$ with total instrument pixel counts around 10,000 . TESs have also benefitted from dedicated programs to improve the sensitivity (reduce the NEP) for space spectroscopy. These programs have demonstrated NEPs as low as $1 \times 10^{-19} \mathrm{~W} \mathrm{~Hz}^{-1 / 2}$ at both Jet Propulsion Laboratory (JPL) and Space Research Organization of the Netherlands. ${ }^{37,38}$ Pushing to lower NEPs is possible though potentially cumbersome: either base temperatures must be reduced from the canonical $50 \mathrm{mK}$, and/or the leg isolation needs to be improved with more exotic microfabricated structures. Another challenge with a TES system in light of the formats required for OSS is the complexity of focal plane assembly, in particular the hybridization with superconducting quantum interference devices (SQUIDs). For both the workhorse time-domain multiplexer ${ }^{39}$ and the newer microwave multiplexer, ${ }^{40,41}$ a SQUID is required for every detector pixel, a challenge for the large OSS arrays with their small pixel pitch. An alternative approach uses a single SQUID at MHz frequencies to read out 100 to 200 detectors, each detector coupled to an $L C$ resonator. ${ }^{42-44}$ This latter approach is promising but also requires advances in resonator packaging and hybridization to scale up to OSS array formats. Finally, we note that the speed of TES bolometers may be a concern when pushed to the lowest NEPs, since fundamentally, the time constant scales as the thermal conductance $G$, and $G$ must be reduced as the square of desired NEP improvements $(\mathrm{NEP} \propto \sqrt{G})$. Khosropanah et al. ${ }^{38}$ indicated time constants of 0.2 to $0.3 \mathrm{~ms}$ for their low-NEP devices, which indicates that $3 \mathrm{~ms}$ is possible at the NEP target for OSS.

Kinetic inductance detectors (KIDs). ${ }^{45}$ KIDs offer a much simpler implementation by combining the detection with the readout resonator, so that in many cases KID arrays can be implemented with one or two thin film metal layers simply patterned on silicon. KIDs have progressed rapidly since their inception in $2003 .^{46}$ They have been and are in use in several ground-based experiments ${ }^{47-52}$ and have recently flown on the Olimpo and BLAST-Pol2 balloon experiments, ${ }^{53}$ performing well. The best reported KID sensitivities are now $3 \times 10^{-19} \mathrm{~W} \mathrm{~Hz}^{-1 / 2}$ (a figure that far exceeds the requirements for ground-based or suborbital work), and this has been demonstrated in at the kilo-pixel formats with good yield and uniformity ${ }^{54}$ The principal challenge for KIDs is to bring down the NEP a further factor of 10 to the OSS target. The most 
straightforward path to lower NEP is to reduce the device volume, and per-pixel area, while maintaining a reasonable resonant frequency and a design that can efficiently absorb photons. An approach based on combining high-quality aluminum with small-volume lumped-element designs $^{55}$ is promising and works to demonstrate that are underway.

Speed is not a problem since KIDs are intrinsically fast with a fundamental limit given by the quasiparticle recombination time, a parameter that is dependent on the material and is important for the sensitivity (longer is more sensitive). It is on the order of a millisecond for the best aluminum films, thus the devices can readily meet the OSS 3-ms requirement.

Quantum capacitance detector $(Q C D) .{ }^{56}$ The QCD is an emerging technology with roots in quantum-computing devices. As with a KID, the incident photons break Cooper pairs and establish a density of quasiparticles (free electrons) in a superconducting absorber, and as with a KID, the device uses radiofrequency (rf)/microwave resonators for multiplexed readout. Unlike a KID, the QCD absorber and resonator are separate elements, coupled through tunnel junction, so there is an additional degree of freedom in the design. Single quasiparticle tunneling events change the capacitance of the resonator enough to shift the frequency by a large fraction of a linewidth, so are readily detectable. Because a single far-IR photon produces several quasiparticles, each of which tunnels many times, the system can easily provide shot-noise-limited performance at very low loading levels. Optical NEPs down to $10^{-20} \mathrm{~W} \mathrm{~Hz}^{-1 / 2}$ have been demonstrated. ${ }^{57}$ As with the KIDs, the devices are intrinsically fast, limited fundamentally by quasiparticle recombination. In fact, the QCD has shown the combination of speed and sensitivity in a far-IR photon counting mode. ${ }^{58}$ The principal challenges for the QCD are to push to larger arrays, to demonstrate the uniformity and stability, and to understand the sensitivity when operated at the upper end of the required OSS dynamic range.

\subsection{Readout Electronics}

All three of the detector approaches for OSS (as well as for the Origins far-IR imager/polarimeter (FIP)) will employ an rf/microwave frequency-domain multiplexing readout in which each pixel is coupled to a narrow-band $\left(Q \sim 10^{5}\right)$ superconducting resonator. The warm electronics generates a comb of frequencies, typically one per pixel, and this comb interacts with the array and returns to the warm electronics. Each tone incurs a frequency or amplitude shift as it interacts with its corresponding detector pixel. The returning comb signal is amplified in the cold with two stages of HEMT (high-electron mobility transistor) amplifiers, one at $4 \mathrm{~K}$ and one at $\sim 35 \mathrm{~K}$. In the warm electronics, it is then digitized and demodulated to extract each frequency's information. No fundamentally new algorithms or approaches are required; it is just a question of matching capability with the Origins system resources.

The readout system for OSS and FIP places demands on the Origins system in two ways: the total electrical power budget (for the processing of the readout datasteam in the warm spacecraft) and on the 4-K cooling budget (for both amplifier dissipation and conducted loads through the coaxial lines). At present, the 4-K dissipation of the readout system is the primary limitation on total pixel count. Up to $2 \times$ more pixels could be accommodated if readout dissipation is reduced, and/or as the current $100 \%$ margin on cooling capability is relaxed as the design matures.

For the OSS point design, we adopted the case with the greatest system-level impact: TES bolometers with the microwave MUX ${ }^{59,60}$ Figure 6 shows the approach. The baseline resonator frequencies are 4 to $8 \mathrm{GHz}$, and the resonator spacing is $2 \mathrm{MHz}$. Each readout circuit, therefore, can process 2000 pixels, and when partitioned into integer readout circuits for the six arrays, the total number of circuits required for all six is 32 . The KIDs and possibly QCDs will also likely operate at $\sim 2000$ pixels per circuit, but will likely use lower readout frequencies (e.g., 0.5 to $2 \mathrm{GHz}$ ). This could translate to lower required power dissipation in the cryogenic amplifiers, allowing more circuits and larger arrays.

The warm side approach is described more fully in the Origins Technology Development Plan. The baseline uses the new Xilinx rf-system on chip (SoC), ${ }^{61}$ which integrates the analog to digital converters (ADC) with programmable digital signal processing logic [floating point gate array (FPGA)-like] in a single chip based on 16-nm-gate complementary metal-oxide semiconductor transistors. A single chip provides eight channels, each capable of processing $2 \mathrm{GHz}$ at 12 bits depth, and the associated waveform generation capability. When compared with our 


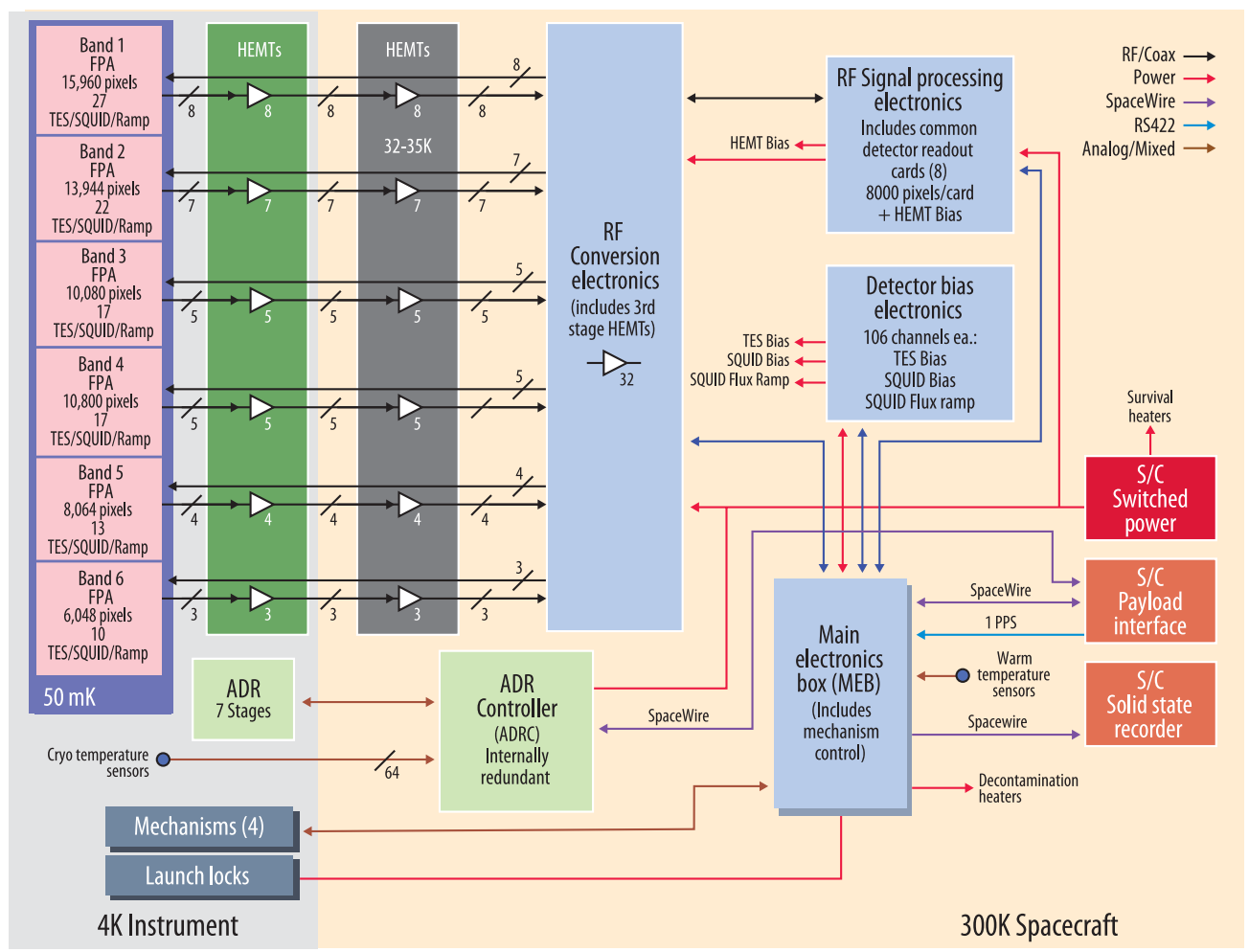

Fig. 6 The readout system consists of 32 circuits, each carrying $4 \mathrm{GHz}$ of information bandwidth. Detectors are coupled to microresonators, 2000 of which can be read out in each line. On the warm spacecraft side, electronics create the waveform that interacts with the array, and then digitizes and analyzes the return waveform to extract phase shifts that encode the optical power on the detector. This basic approach accommodates all viable detector technologies under the development for Origins.

existing $500 \mathrm{MHz}(0.5 \mathrm{GHz})$ single-channel FPGA-based systems now in use, the new rfSoC represents $\sim 8 \times(2 / 0.5 \mathrm{GHz})=32$ times the processing capability. Abaco released a board with this chip in late 2018. Initial power dissipation estimates for this board are $50 \mathrm{~W}$ at full utilization.

For the system design, OSS uses this power dissipation fiducial of $50 \mathrm{~W}$ per $16 \mathrm{GHz}$ of information bandwidth for the ADC and digital spectrometer logic. This is a conservative estimate-while neither of these existing systems are flight-qualified, mixed-signal applicationspecific integrated circuits, which integrate ADCs and signal processing, have been developed for flight implementation of similar applications, ${ }^{62}$ and these systems typically have lower power dissipation than their programmable-logic counterparts.

\subsection{Detector Technology Development Plan}

The heritage of the OSS detector system and its path to flight readiness is detailed in the Origins Technology Development Plan, ${ }^{63}$ a supplement to the full report. In this, we propose a 7-year program beginning in 2021 designed to dovetail with the flight implementation program beginning around 2025. The program serves both OSS and the imager-polarimeter FIP. It will achieve technology readiness level (TRL)-5 (performance at representative array/multiplexing scale) in 2025 and TRL-6 (with environmental qualification) in 2027. The strategy is to pursue two technology paths, TES bolometers, and KIDs, and to also fund promising lower TRL technologies such as the QCDs with $\sim 20 \%$ of the program budget. This approach is chosen to mitigate mission risk of inadequate detector development in time for mission implementation. Unlike a traditional grant-based program, we will implement a review and progress assessment program, so that a technology that meets Origins requirements can be chosen as early as possible. For example, if during the initial phases of the program, one of the lower-TRL technologies takes off 
and appears more likely to achieve the requirements than KIDs or TES bolometers, then resources can be redirected to maturing that technology.

\subsection{Data Rate}

Far-IR direct detectors must be sampled continuously, and the OSS data rate is determined by the pixel count $(60,000)$, sampling rate, and required bit depth. The detectors will be sampled at $200 \mathrm{~Hz}$ to support an electrical bandwidth of $100 \mathrm{~Hz}$, a comfortable factor of 2 above the $50-\mathrm{Hz} 3-\mathrm{dB}$ frequency of the detector with its 3-ms time constant. 12 bits is sufficient to capture the detector's dynamic range under which it is photon noise limited if a logarithmic scaling is used. The data rate to ground without any compression is thus $144 \mathrm{Mbits} / \mathrm{s}$ (where 1 Mbit $=1,000,000$ bits), a figure which can be accommodated by the emerging optical communication systems. In the high-resolution modes, since only a small fraction of the array is used, the rate could be smaller, but we expect that the grating data may be valuable for other purposes during these observations (e.g., for monitoring background and taking potentially serendipitous measurements along the slit).

\section{Expected Performance}

Figure 7 shows the expected OSS low-resolution sensitivity in the far-IR and submillimeter as well as the spectral survey speed. The OSS instrument is the suite of $R=300$ grating with the slit lengths as designed (Table 1)

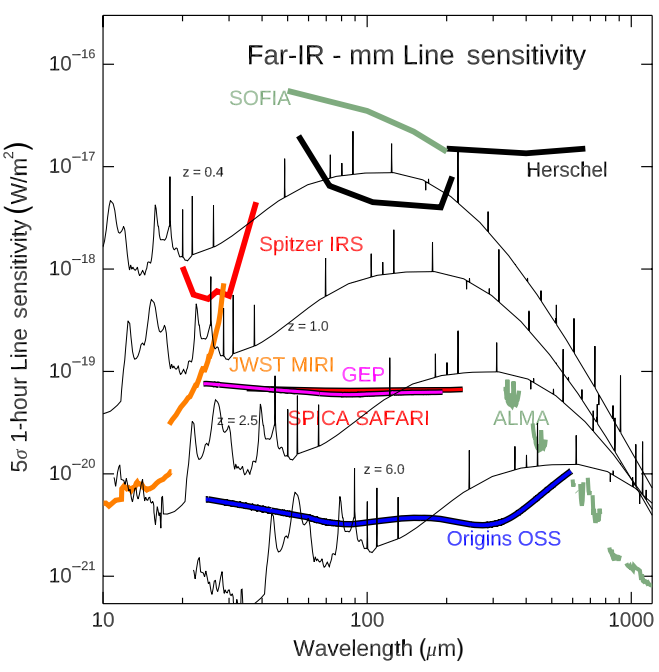

(a)

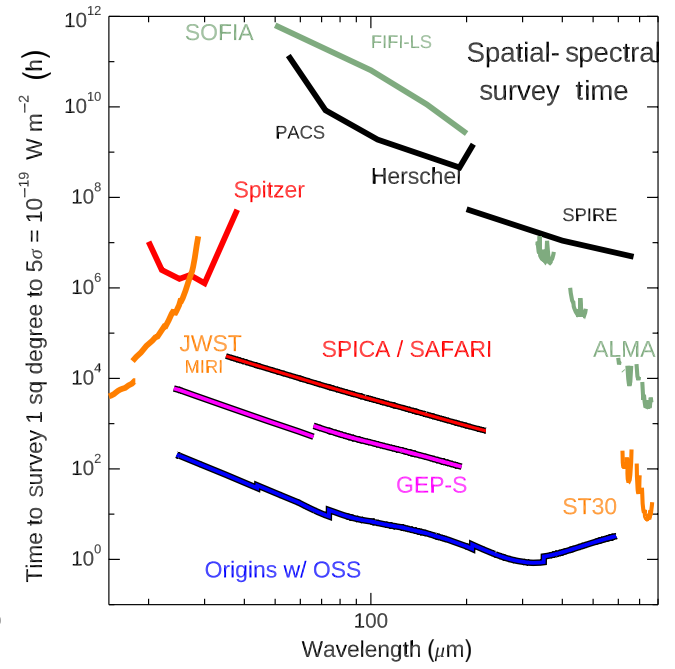

(b)

Fig. 7 OSS grating mode sensitivity. (a) The sensitivity in $\mathrm{Wm}^{-2}$ for a single pointed observation, including a conservative assumption about background subtraction (see text). OSS covers the full 25 to $588 \mu \mathrm{m}$ band, simultaneously. The SPICA/SAFARI-G ${ }^{7,8}$ curve refers to the studied configuration: a 2.5-m telescope with a suite of $R=250$ grating spectrometer modules with 5 spatial beams, and detectors with NEP $=2 \times 10^{-19} \mathrm{~W} \mathrm{~Hz}^{-1 / 2}$ but no other margin (expect detector NEP around $\left.1 \times 10^{-19} \mathrm{WHz}^{-1 / 2}\right)$. GEP ${ }^{9}$ denotes the galaxy evolution probe concept, a 2-m cryogenic telescope with a suite of $R=200$ wideband spectrometers with 40 to 70 beams, and a detector NEP of $1 \times 10^{-19} \mathrm{WHz}^{-1 / 2}$. ALMA sensitivity refers to an $R=1000(300 \mathrm{~km} / \mathrm{s})$ bin, and the survey speed incorporates the number of tunings of the $16 \mathrm{GHz}$ total bandwidth to cover a $1: 1.5$ fractional band. Galaxy spectra assuming $L=10^{12} L_{\odot}$ at various redshifts are overplotted using light curves (with continuum smoothed to $R=300$ ). (b) The time for a blind spatial-spectral survey reaching a line-flux depth $(5 \sigma)$ of $10^{-19} \mathrm{~W} \mathrm{~m}^{-2}$ over a square degree. This survey time metric scales as the sensitivity squared over the instantaneous field of view (slit width $\times$ length for OSS), and for large maps, background subtraction should be a negligible effect. This shows the 6 to 10 orders of magnitude speed advance available with Origins OSS for survey measurements. 


\subsection{Sensitivity Model}

The relationship between the detector performance and the estimated on-sky sensitivity are similar to what has been obtained in ground-based grating spectrometers Z-Spec ${ }^{64}$ and ZEUS, ${ }^{35,65}$ providing confidence in the estimation. The model assumes a 5.9-m-diameter telescope with $72 \%$ aperture efficiency to a point source, and $25 \%$ total instrument transmission (in a single polarization) for the base low-resolution mode. Detectors are assumed to operate with $\mathrm{NEP}=3 \times 10^{-20} \mathrm{~W} \mathrm{~Hz}^{-1 / 2}$, a figure which has been demonstrated in the lab with the QCD. The line-detection sensitivity includes a factor of $\sqrt{N_{\text {eff }}}$, the effective number of pixels used to optimally extract the line flux. For the OSS grating modules, which have detectors sampling and the spectrometer intrinsic resolving power comparable at $\mathcal{R}=300, N_{\text {eff }} \sim 1.6$, this adds a factor of 1.26 .

\subsubsection{Sensitivity margins}

In addition to the factors noted above, the sensitivity estimates include two forms of margin: (1) the model includes a factor of 1.7 above the calculated sensitivities values assuming a total slit-to-detector efficiency of $25 \%$, this can allow for a combination of lower efficiency and/or poorer detector sensitivity. (2) The point-source staring sensitivity (which employs chopping of the Origins FSM) includes a factor of $\sqrt{2}$ penalty. This would be required if the source is only observed half the time or if the background is only measured with a single spatial mode. But since the baseline approach is to chop the source along the slit and measure the background with the full slit, this factor does not apply in the current design, so it represents margin. Additionally, we note that as part of the Origins study, all science cases and time estimates are based on a performance that is $2 \times$ poorer than the calculated sensitivity values, which themselves include the factors described above.

\subsubsection{Detector sensitivity}

The target detector NEP for OSS is $3 \times 10^{-20} \mathrm{~W} \mathrm{~Hz}^{-1 / 2}$, but because the system is strongly background limited at this value, some degradation in detector sensitivity can be accommodated without greatly impacting sensitivity. Although the sensitivity depends on wavelength and sightline through the zodiacal dust and cirrus dust, Fig. 5 shows an example calculation for the North Ecliptic pole at $100 \mu \mathrm{m}$. Here, a fallback in the detector NEP from the target to a much easier $1 \times 10^{-19} \mathrm{~W} \mathrm{~Hz}^{-1 / 2}$ (already demonstrated with TES bolometers by multiple groups) results in only a factor of 1.8 degradation in sensitivity. More typical sightlines will see smaller effects.

\subsection{Bright Sources and High-Resolution Modes}

Further applications of the sensitivity model are shown in Fig. 8. Bright sources add shot noise and degrade the sensitivity in a straightforward manner. For spectroscopy of solar-system objects, OSS must permit observations of sources up to $5 \mathrm{Jy}$ at $128 \mu \mathrm{m}$ without saturation. This translates to a dynamic range from the Zodi background loading of 1000, a requirement on the detector system.

For the high-resolution modes, the estimated sensitivities include the additional signal loss (52\%) and loading from the 4.5-K FTS optics, and another factor of 0.25 transmission through the etalon (49\% due to Ohmic loss in the mirrors $\times$ an estimated $75 \%$ due to beam walk-off in the large cavity). The very-high-resolution mode requires stepping the etalon to create a full spectrum, an additional sensitivity factor that is not included in the curves in Fig. 8.

\section{Thermal and Mechanical Design and Resource Requirements}

Table 3 summarizes the top-level resource requirements imposed by the OSS on the observatory. Mass estimates carry contingency and margin from the current-best-estimate (CBE) values; structures are designed to accommodate these margined values. 
Bradford et al.: Origins Survey Spectrometer: revealing the hearts of distant galaxies...

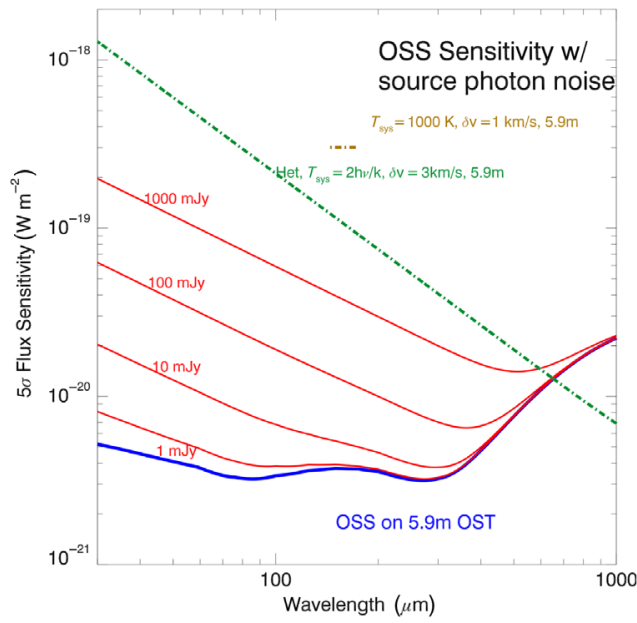

(a)

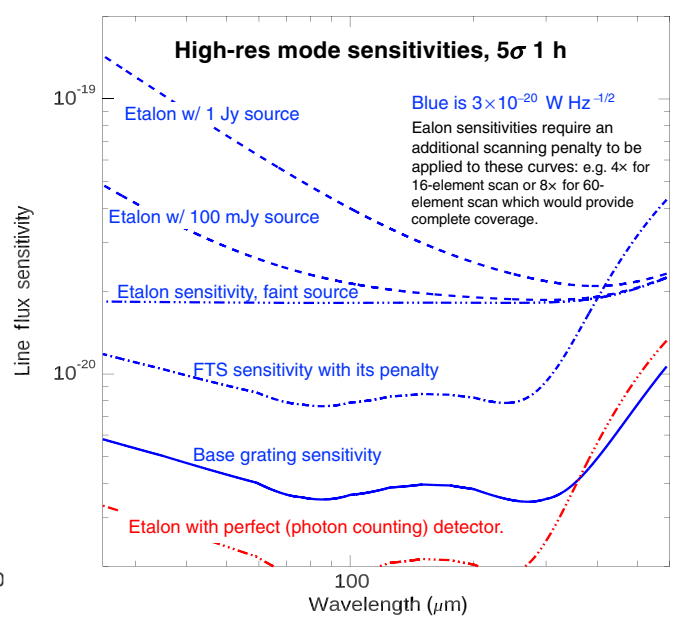

(b)

Fig. 8 (a) Sensitivity degradation of the base grating mode in the face of photon noise from bright sources. Also plotted for comparison are sensitivities possible with possible optimized heterodyne receivers operating near the quantum limit (dark green) and with $T_{\text {sys }}=1000 \mathrm{~K}$ (brown), assuming a linewidth of $3 \mathrm{~km} / \mathrm{s}$. (b) The high-resolution mode sensitivities. The FTS has a sensitivity penalty due to on-source time and losses. The etalon compounds this with additional losses and the etalon must scan, a factor not included in these plots. The etalon is baselined to cover the 100- to $200-\mu \mathrm{m}$ range, with coverage over the full band as a goal, but achieving the etalon sensitivity beyond $200 \mu \mathrm{m}$ will require careful attention to stray light from the instrument itself.

Table 3 Top-level resources for OSS

\begin{tabular}{|c|c|c|c|}
\hline \multicolumn{2}{|l|}{ Attribute } & \multirow{2}{*}{$\frac{\mathrm{CBE}}{624}$} & \multirow{2}{*}{$\frac{\mathrm{CBE}+\text { contingency }+ \text { margin }}{889}$} \\
\hline Mass (kg) & Total & & \\
\hline & Interferometer (including bench) & 193 & \\
\hline & Grating spectrometers (incl. structure, arrays) & 190 & \\
\hline & ADR and thermal straps & 25 & \\
\hline & Miscellaneous cold mounting and fixturing & 54 & \\
\hline & Wiring harnesses, cold amps & 86 & \\
\hline & Warm electronics and housing & 77 & \\
\hline \multirow[t]{3}{*}{ Power (W) } & Average in operation & 558 & 725 \\
\hline & Peak in operation & 945 & 1228 \\
\hline & Standby/safehold & 188 & 244 \\
\hline \multirow[t]{2}{*}{ Data rate } & Readout, raw & 1.5 Tbps & $\mathrm{n} / \mathrm{a}$ \\
\hline & Science data (Mbps) & 146 & 190 \\
\hline \multicolumn{2}{|c|}{ Heat rejection to $4 \mathrm{~K}(\mathrm{~mW})$} & 26 & 63 \\
\hline
\end{tabular}

Note: Mass is allocated a $14 \%$ contingency plus $25 \%$ margin (held at observatory level). Power and data rate are allocated $30 \%$ margin at system level. 


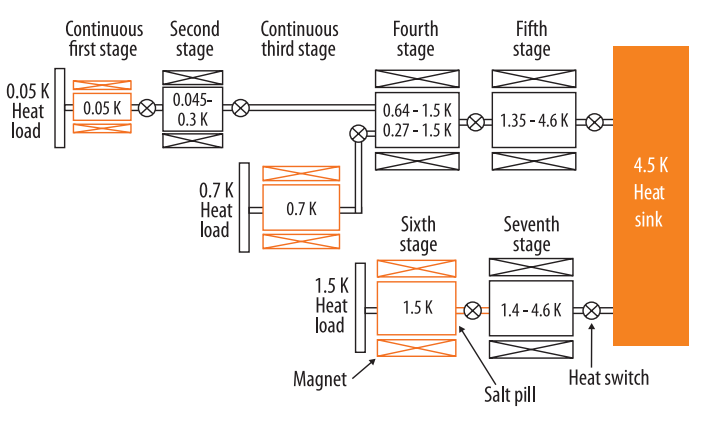

(a)

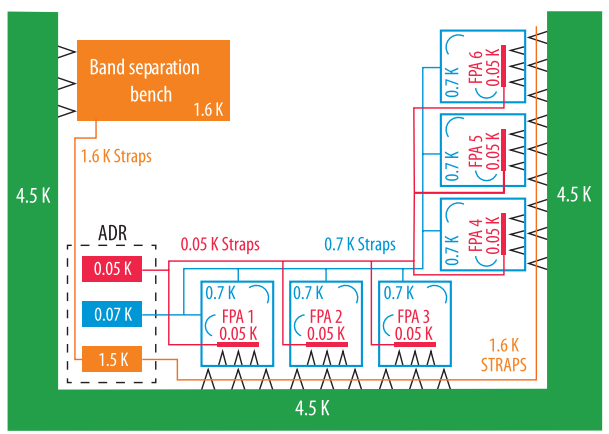

(b)

Fig. 9 OSS thermal architecture. (a) The seven-stage continuous ADR is actually a five-stage ADR, similar to that used in the FIP instrument, and a two stage ADR. The ADRs are controlled from a single ADR controller on the warm side of the spacecraft. (b) Low-thermal-conductivity titanium bipods mount the spectrometer boxes from the optical bench, and the focal plane assemblies inside the spectrometers. These comfortably support launch loads, yet enable the 50 -mK cryogenic system; no special provisions are required to survive launch. Though numerous, the cooling straps from the ADR are the size of large gauge copper wires and weigh a combined total of $1 \mathrm{~kg}$.

\subsection{OSS Thermal Design}

The interferometer optics operate at the observatory-provided base temperature of $4.5 \mathrm{~K}$, but the grating modules as well as the band separation elements (polarizing grid and dichroic filters) are all cooled further to ensure that their thermal emission does not impact sensitivity. In particular, the potentially lossy grid and dichroic filters before the grating slits (termed the band separation optics) must be cooled to below the microwave background temperature to ensure that they do not add background. The gratings modules themselves need to be below $0.9 \mathrm{~K}$-at least at the long wavelengths - to ensure that the integrated power through the bandpass filter with the full native detector étendu $(\mathrm{A} \Omega)$ does not saturate the detector or degrade the noise equivalent power. These requirements are met with a continuous adiabatic demagnetization refrigerator (CADR), which stages from the observatory-provided $4.5-\mathrm{K}$ system. A schematic is provided in Fig. 9. The CADR is adapted from the high-heritage Goddard Space Flight Center (GSFC) designs of the Hitomi ADR and subsequent technology advances. ${ }^{66-68}$ It uses 7 salt pills to provide 3 continuously-cooled stages: at $1.5 \mathrm{~K}$ (for the polarizing grid and dichroic filters), $0.7 \mathrm{~K}$ (for the grating spectrometer enclosures), and $0.05 \mathrm{~K}$ (for the focal plane arrays themselves). A key advantage of the ADR approach is the high Carnot efficiency, and the CADR heat rejection is only about half of the $4.5-\mathrm{K}$ budget even though it is designed to provide $100 \%$ lift margin (that is $2 \times$ what is needed) at all of its actively cooled stages. Further information is provided in the Origins Technology Development Plan. A suspension system has been designed together with the cooling system; its schematic is included in Fig. 9. The band separation optics and the grating optics modules are suspended kinematically from $4.5 \mathrm{~K}$ with bipods made of titanium 15-3-3-3, a low-thermal-conductivity alloy. Although composites offer lower thermal conductance at these temperatures, the use of metal eliminates a potential source of water contamination curing cool down. For the grating optics modules cooled by the $0.7 \mathrm{~K}$ stage, the titanium supports couple directly from $4.5 \mathrm{~K}$ mechanically, but heat is intercepted at the strut midpoints by the 1.6-K stage. Strut dimensions are tuned for each band's mass, ensuring a resonant frequency that satisfies a standard mass acceleration curve. Inside each grating module, the focal plane assemblies are also mounted kinematically with Ti-15-3-3-3 bipods, in this case designed to provide resonant frequencies of at least $100 \mathrm{~Hz}$.

Table 4 summarizes the calculated heat load and available lift with the cooler for each of the three OSS cooling stages. The dominant load on the sub-4K OSS-cooled stages is the conducted loads through the mechanical suspension. Wiring parasitics are negligible because superconducting cables are used. The $4.5-\mathrm{K}$ situation is more challenging relative to the adopted allocation from the observatory, as this stage must accept the loads from the nonsuperconducting harnesses, and $4.5 \mathrm{~K}$ houses the first stage low-noise amplifier. The $0.38-\mathrm{mW}$ per amplifier $\times 31$ amplifiers correspond to commercially available devices operating at up to $8 \mathrm{GHz}$, but the team expects 
Bradford et al.: Origins Survey Spectrometer: revealing the hearts of distant galaxies...

Table 4 OSS cryogenic stages: thermal loads and available lift

\begin{tabular}{|c|c|c|c|c|c|}
\hline$T(\mathrm{~K})$ & Source & Heat load & Total & Capability & Margin (\%) \\
\hline \multirow[t]{3}{*}{0.05 (focal planes) } & Harness conducted & $0.65 \mu \mathrm{W}$ & $2.67 \mu \mathrm{W}$ & $6.0 \mu \mathrm{W}$ & 125 \\
\hline & Dissipation & $0.67 \mu \mathrm{W}$ & & & \\
\hline & Suspension conducted & $1.35 \mu \mathrm{W}$ & & & \\
\hline \multirow[t]{2}{*}{0.7 (grating modules) } & Harness conducted & $6.5 \mu \mathrm{W}$ & $111 \mu \mathrm{W}$ & $292 \mu \mathrm{W}$ & 163 \\
\hline & Suspension conducted & $104 \mu \mathrm{W}$ & & & \\
\hline \multirow[t]{3}{*}{1.5 (bench and intercepts) } & Harnesses conducted & $0.04 \mu \mathrm{W}$ & $2.11 \mathrm{~mW}$ & $4.24 \mu \mathrm{W}$ & 101 \\
\hline & Bench supports conducted & $0.96 \mathrm{~mW}$ & & & \\
\hline & FPA supports & $1.11 \mathrm{~mW}$ & & & \\
\hline \multirow[t]{3}{*}{4.5 (from observatory) } & Amplifier dissipation & $11.8 \mathrm{~mW}$ & $26 \mathrm{~mW}$ & $63 \mathrm{~mW}$ & 144 \\
\hline & Mechanisms & $1 \mathrm{~mW}$ & & & \\
\hline & ADR heat rejection & $13 \mathrm{~mW}$ & & & \\
\hline
\end{tabular}

improvements in amplifier power dissipation will be possible by the time Origins is built. A straightforward descope to ease this aspect is to not operate all six bands simultaneously. (Parasitic conduction in harnesses is book-kept at observatory level, but is much smaller than the amplifier dissipation.) The design includes thermal straps made of pure annealed copper for all stages, sized for the load, and distance required. These copper straps along with the indium used for thermal connections total $\sim 8 \mathrm{~kg}$ for OSS.

\subsection{Mechanical Approach}

Each of the OSS grating modules and the interferometer bench are discrete optomechanical elements that will be fabricated and tested independently and then mounted kinematically in the full instrument. They can be internally aligned and are subjected to environmental testing as units before being integrated into the full instrument. Aluminum spectrometer optical elements (mirrors and gratings) are the baseline, but the structural benches are baselined as beryllium to increase stiffness and reduce mass.

\subsection{Mechanisms}

OSS has four mechanisms tabulated in Table 5 with their travel and accuracy requirements Me1 is the FTS interferometer scan mechanism that moves the rooftop mirror carriage. The required accuracy is driven by ensuring that in a given sample in the interferogram, the power uncertainty due to OPD error is less than the uncertainty due to the other noise sources (only photon noise in the optimal case). Quantitative assessment requires simulations, as was done for Herschel SPIRE, ${ }^{69,70}$ a subject for phase-A study. But a key point is that for random errors in OPD, the required accuracy scales as $\lambda$ and the ratio $(\mathrm{NEP} / \sqrt{\tau}) / P$. With its lower background, the latter ratio is more than $4000 \times$ greater for OSS than for SPIRE for a given sample time. We, therefore, expect random errors will be much less stringent for OSS than for SPIRE, even with the shorter wavelengths. Pending the simulation work, we adopt for the purposes of the study a value of $\lambda / 4$ in OPD, which is more stringent than the scaling would suggest. This translates to $0.8 \mu \mathrm{m}$ in physical displacement with the $8 \times$ path folding. In addition to verifying this, a key subject for phase-A simulation work is to assess the requirements on nonrandom (systematic) errors. These will have to be controlled to a greater level to avoid impacting long observations. The angular runout requirement is given by the need to maintain good pupil overlap in the interferometer over its full scan length. 
Bradford et al.: Origins Survey Spectrometer: revealing the hearts of distant galaxies...

Table 5 OSS mechanism requirements

\begin{tabular}{|c|c|c|c|c|}
\hline$\#$ & Purpose & Motion & Accuracy & Approach \\
\hline Me1 & FTS scanning & $\begin{array}{l}\text { Move } 20 \mathrm{~kg} \text { mass: } \\
\text { (a) } 100 \mathrm{~mm} \text { at } 10 \mathrm{~mm} / \mathrm{s} \\
\text { and (b) } 300 \mathrm{~mm} \text { at } \\
300 \mu \mathrm{m} / \mathrm{s}\end{array}$ & $\begin{array}{l}0.8 \mu \mathrm{m} \text { knowledge, } \\
1.6 \mu \mathrm{m} \text { control, angular } \\
\text { runout of } 1.6 \text { arc sec }\end{array}$ & $\begin{array}{l}\text { Multi-arm flex-band (Fig. 10) } \\
\text { with superconducting linear } \\
\text { servo }\end{array}$ \\
\hline $\mathrm{Me} 3$ & Etalon fine scan & $\begin{array}{l}\text { Move one etalon mirror } \\
0.5 \mu \mathrm{m} \text { every } 10 \mathrm{~s} \\
\text { total throw of } 600 \mu \mathrm{m} \text {. }\end{array}$ & $\begin{array}{l}0.2 \mu \mathrm{m} \text { knowledge and } \\
0.4 \mu \mathrm{m} \text { control. Angular } \\
\text { runout }<0.3 \text { arc sec }\end{array}$ & $\begin{array}{l}\text { Piezoelectric inchworm; } \\
\text { position via Kaman } \\
\text { differential impedance } \\
\text { transducer or Mad City } \\
\text { Labs NanoAlign-3 }\end{array}$ \\
\hline Me2 & $\begin{array}{l}\text { FTS pickoff } \\
\text { engagement }\end{array}$ & $\begin{array}{l}\text { Move FTS pickoff and } \\
\text { reinsertion mirrors into } \\
\text { beam }\end{array}$ & $\begin{array}{l}\text { Position to } \sim 1 \mathrm{~mm} \text {, } \\
\text { oriented to within } \\
20 \text { arc sec }\end{array}$ & $\begin{array}{l}\text { 4-bar linkage w/brushless } \\
\text { dc motor }\end{array}$ \\
\hline Me4 & Etalon insertion & $\begin{array}{l}\text { Move etalon assembly } \\
\text { into inteferometer beam }\end{array}$ & $\begin{array}{l}\text { Translation control to } \\
2 \mathrm{~mm} \text {, oriented to } \\
10 \text { arc sec }\end{array}$ & $\begin{array}{l}\text { 4-bar linkage w/brushless } \\
\text { dc motor }\end{array}$ \\
\hline Me-L & \multicolumn{2}{|c|}{ Launch locks for the above } & $\mathrm{N} / \mathrm{A}$ & $\begin{array}{l}\text { Non-explosive actuator, } \\
\text { e.g., NEA } 9100\end{array}$ \\
\hline
\end{tabular}

Me2 is for the interferometer insertion; it simply moves the carriage containing the pickoff and reinsertion mirrors into the beam. Position accuracy is not a strong constraint, but this carriage must have an orientation accurate to $20 \mathrm{arc} \mathrm{sec}$ to ensure the interferometer pupil matches the telescope pupil. Me3 scans the etalon, it needs only cover a couple $\times$ the free spectral range (FSR) at the longest wavelength $\left(\mathrm{FSR}=\lambda_{\max } / 2\right)$, so a total of $600 \mu \mathrm{m}$. However, it must provide translation knowledge to $0.2 \mu \mathrm{m}, \sim 1 / 4$ of a resolution element at $112 \mu \mathrm{m}$. Furthermore, it must maintain parallelism to $0.3 \mathrm{arc} \mathrm{sec}$ to ensure that the cavity finesse is not impacted. Finally, Me4 allows the etalon assembly to be inserted and removed from the interferometer beam. Here, the positional accuracy is not critical, but the etalon must be oriented to within 10 arc sec once inserted to avoid beam walk-off in the etalon. The FTS and etalon scan mechanisms and the associated lubricants are specified for a minimum of 10 years and one million cycles, ample to accommodate any conceivable combination of observations over Origins' mission life. The insertion mechanisms for the FTS pickoff and etalon are specified for 2000 cycles, a figure which is likely ample, but which should be revisited in phase-A as observing scenarios become clearer.

Detailed mechanism designs will be undertaken in phase A, but Fig. 10 shows an approach that meets the FTS translation requirements and was used in the study. This dual-stage parallelogram flex-band type system has essentially no friction. The pictured prototype was developed at NASA Goddard ${ }^{71}$ for use in Herschel SPIRE; ${ }^{69}$ it will be scaled up by a factor of 3 to achieves the required OSS linear travel of $300 \mathrm{~mm}$. A superconducting linear servo motor has the required precision and low losses allowing for a dissipation of $<1 \mathrm{~mW}$. For the etalon, a piezoelectric inchworm actuator will be used. Position, to the accuracy required, will be read out with either a modified Kaman DIT ${ }^{72}$ or a modified Mad City Labs NanoAlign- $3{ }^{.73}$ both product families have some flight heritage. The launch locks will be actuated using typical nonexplosive actuators such as the NEA 9100. For the etalon and FTS insertion mechanisms, which are used infrequently, a four-bar linkage connected to a brushless dc motor is used.

\section{Instrument Control}

The OSS control schematic is shown in Fig. 11. Although the OSS is large and has two powered spectroscopic modes, each motion requires only a single degree of freedom actuation with position encoded, similar to what was used for the Herschel SPIRE spectrometer. ${ }^{26}$ Similarly, while the detector technology is different, the detector bias and readout at a high-level function similar to those of the bolometer systems in SPIRE and the high-frequency instrument on Planck. ${ }^{74,75}$

J. Astron. Telesc. Instrum. Syst. $\quad$ 011017-18 Jan-Mar 2021 • Vol. 7(1) 


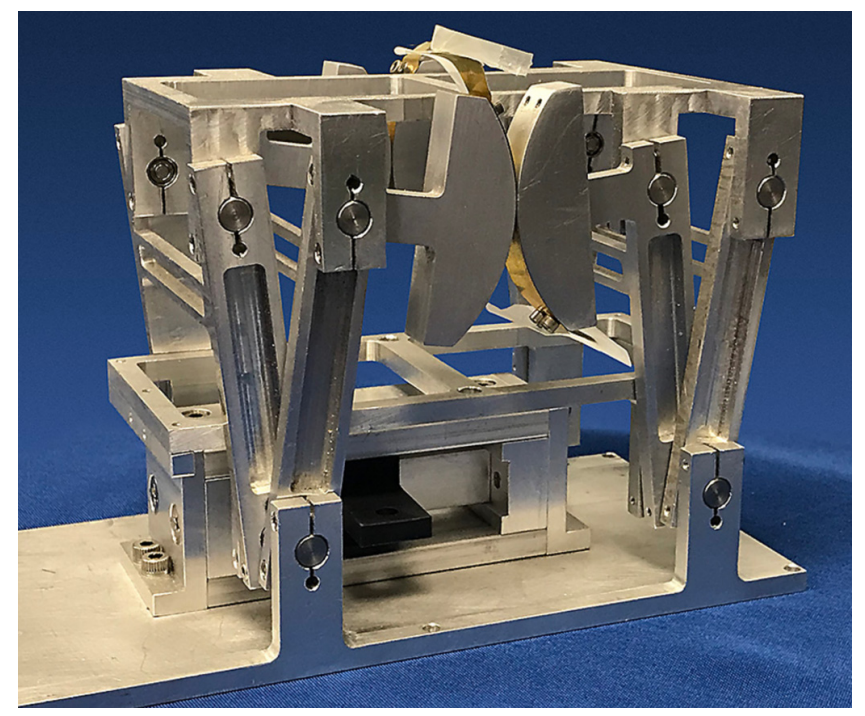

Fig. 10 Flex type mechanism for use with the OSS FTS. This mechanism is free of friction, can be operated in any orientation, and has a stroke of $10 \mathrm{~mm}$. For OSS, this would be scaled up by a factor of 3 .

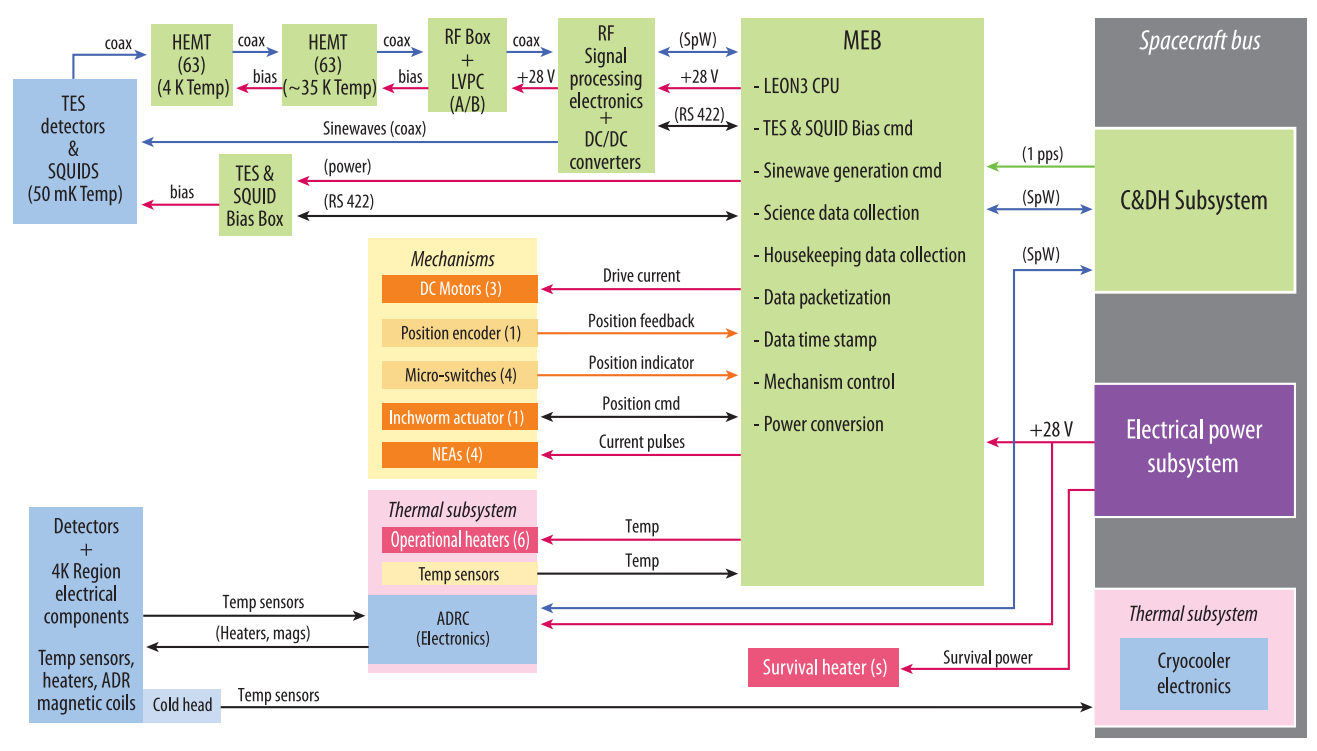

Fig. 11 Block diagram of OSS instrument control architecture. HEMT refers to the cryogenic microwave amplifiers (high-electron mobility transistor), and LVPC stands for low-voltage power converter used to bias the amplifiers. The MEB houses the CPU, detector bias and acquisition electronics, and control electronics for the mechanisms. Given that the modes are built up primarily from pre-existing elements (e.g., FTS scan), the software reuse is estimated at $85 \%$.

They are steady-state systems, collected passively, with no real-time decision-making or analysis required on the spacecraft. The system is thus in family with SPIRE and other astrophysics instruments with a modest number of discrete mechanisms. The baseline design has a main electronics box (MEB), which includes a number of specialized control boards and a LEON3 CPU. The system includes all critical features as follows.

- Mode management (allowing reboots with software updates, standby, calibration, and the various science observing modes).

- Instrument support (command processing, data collection, and support for firmware updates). 
- Mechanism control. FTS and etalon scan are operated with closed-loop control with encoder feedback at $1 \mathrm{kHz}$. Interferometer insertion and etalon insertion are open-loop commanded, with positions verified by microswitches.

- Power distribution.

- Some degree of on-board autonomy: limit checking, some processing command sequences, failure corrections.

Flight control software for the OSS is straightforward, and we have identified no technical risks. It will be a real-time system based on proven JPL or GSFC in-house or commercial off-theshelf systems. The system will have an estimated 60,000 total lines of code of which $\sim 85 \%$ will be reused from existing flight instruments, based on the similarity of the individual elements of the OSS system (individual mechanisms, high-level data acquisition and storage, and housekeeping) to those used on other flight instruments such as SPIRE. As discussed in Sec. 5.1, new elements required for the microwave multiplexing (waveform generation, analog-to-digital conversation, and digital demodulation) will be handled in dedicated programmable logic fabric on the $\mathrm{rfSoC}$ and are outside of processor and flight software. We estimate that the system has 50\% processor margin with the LEON-3 CPU.

\section{Alignment, Integration, Testing, and Calibration}

\subsection{Optical Alignment}

The grating modules can be aligned readily either by the vendor or by instrument team members - the elements within each grating module should be aligned sufficiently just from machining tolerances. A good example of this approach is the aluminum grating spectrometer modules of the Spitzer Infrared Spectrograph (IRS), which had even tighter tolerances due to the shorter wavelengths. An aspect not used on Spitzer, but potentially useful for OSS is the fact that the grating module optics, along with the large, field-filling arrays, allows adjustment of the slit position by several beams with respect to the rest of the optics to obtain a desired slit position relative to the grating modules box. Each slit opening is an independent piece that can be adjusted before being clamped into position. The OSS requires that the six grating modules be positioned so that their slits overlap (field alignment), and that they each have their pupil aligned with the telescope pupil (pupil alignment). Meeting the first requirement consists of simply moving the grating module (or, for small adjustments, moving the slit openings) so that the slits align in position, using a telescope simulator which produces a point-source image. The second requires tipping/tilting the grating modules about the slit positions so that they point to a common pupil in the simulator. These tests will have to be performed with a cryogenic mounting of the grating modules.

Alignment of the interferometer begins with a self-alignment ensuring that the collimated beam remains centered as it traverses the mirrors and retroreflectors, and that the two sides of the interferometer recombine with good overlap. This should be possible to do warm if good practices are used in the machining and assembly of the FTS bench (uniform materials and thermal stress relieving), but it must be verified cold. Aligning the interferometer with the grating system requires positioning the interferometer insertion mirrors to provide an extremely wellcollimated beam to permit the etalon operation. Here, the distance along the optical axis from the telescope is important, and this may require a cryogenic test. The interferometer must then have its pupil aligned with the telescope pupil; this consists of tilting the interferometer about the insertion mirror. Finally, the reinjection mirror must then be adjusted to ensure the interferometer's pupil aligns with those of the gratings. The team expects precision machining of the interferometer insertion/reinjection mirror pair as a single carriage will result in having no need to make adjustments. Ultimately, a cold test must be done to validate the results of these alignment activities. The two key success criteria are: (a) transmission efficiency from the OSS front focus (corresponding to the telescope focus) through the interferometer to the detectors, likely with a chopped thermal load viewed through a small aperture and (b) spectral purity (especially for the etalon) verified with a narrowband source such as a far-IR laser or $\mathrm{THz}$ multipler source.

With this full system aligned in the flight truss, which is brought as a unit to the telescope, a full alignment check of all elements should not be required on the observatory. Simply verifying 
pupil overlap (also known as pupil alignment) of the grating modules with the observatory should be sufficient since the Origins telescope has sufficient actuation authority to adjust the telescope boresight and focus depth with respect to the instrument using actuation at both the secondary (6 degrees of freedom) and FSM (2 degrees of freedom).

\subsection{Test Facilities}

OSS will require cryogenic test facilities, but they will be straightforward and pose no particular technical challenges. The detector arrays and individual grating modules will require a refrigerator with 2-K cooling of volumes on order $40 \times 40 \times 50 \mathrm{~cm}^{3}$, with cold fingers allowing the arrays to cool to $50 \mathrm{mK}$. This will verify basic sensitivity and stray-light rejection at the individual module level. Such systems are available commercially. Examples include the closed-cycle dilution refrigerators made by BlueFors, Oxford, and Leiden Cryogenics. For the multi-spectrometer alignment, a larger cryogenic test is required, simultaneously encompassing $1.5 \mathrm{~m}$ to accommodate the optical bench and at least three spectrometers. However, for this test, the full structure only needs to be cooled to $\sim 30 \mathrm{~K}$, as this is sufficient to incur all coefficient of thermal expansion related alignment effects. Cold fingers are required at $250 \mathrm{mK}$ to allow the detectors to operate in a high-background mode and verify alignment. Full sensitivity is not required. These tests can be carried out in one of the existing thermal-vacuum chambers at JPL or GSFC.

\subsection{Calibration}

In considering calibration uncertainties for measurements where the radiometric signal to noise is sufficient, we expect to achieve $\leq 1 \%$ relative measurement accuracy and $\leq 5 \%$ absolute flux calibration. Every detector pixel in the OSS spectrometer modules will have four key measurements generated in integration and test in a dedicated low-background spectrometer testbed that can house any of the grating modules. (1) Sensitivity will be measured with a temperaturecontrolled slit-filling load (blackbody source). (2) Spectral response described below. (3) Optical efficiency is obtained from the response as the slit-filling load is elevated in temperature, by comparing blackbody curves integrated over the measured bandpasses. (4) Beam shape is measured via an external (to the testbed) chopped warm source rastered in the far field of the grating modules. This is essentially verification to reveal any problems with grating module optics so they can be fixed ahead of full integration. Tests 2 and 4 will couple the grating module to outside the testbed, so will require substantial blocking filters to stay within the dynamic range of the OSS detector arrays - this can be accomplished with a combination of dielectric blockers and resonant mesh filters.

As for Herschel and Spitzer, radiometric scientific calibration is ultimately tied to astronomical sources. Given our experience with Herschel, we do not believe an on-board calibration source is needed, and therefore, the baseline OSS design does not include one. However, we will study this question further in phase A.

A key aspect unique to the grating spectrometer is the need for accurate measurement of the spectral response of every detector. This may reveal low-level grating ghosts and other peculiarities that do not impact sensitivity, but could be important in deep observations of sources with a large spectral dynamic range (very bright lines and faint lines or faint lines on a bright continuum). We will measure spectral profiles with a dynamic range of at least 1000 and a resolving power of at least $3000 \times(100 \mu \mathrm{m} / \lambda)$ using a long-path FTS, with the spectral scale anchored by spectral standards such as absorption-cell measurements and a far-IR laser. Once the OSS interferometer is integrated with the instrument, these profiles can be verified with the OSS FTS.

We do not expect the wavelength calibration and spectral profiles to change from the lab to flight, since there are not moving parts, but the on-board FTS will continue to be a benefit for OSS through the mission, as it will provide long-term tracking of the grating system spectral response in the unlikely event that something changes in a grating module. The etalon wavelength scale will be established during integration with a far-IR laser and/or a narrow-band $\mathrm{THz}$ source and confirmed and periodically monitored on orbit with astronomical line-emitting spectral standards observed on Herschel and Stratospheric Observatory for Far-Infrared Astronomy (SOFIA) such as IRC+10216. 


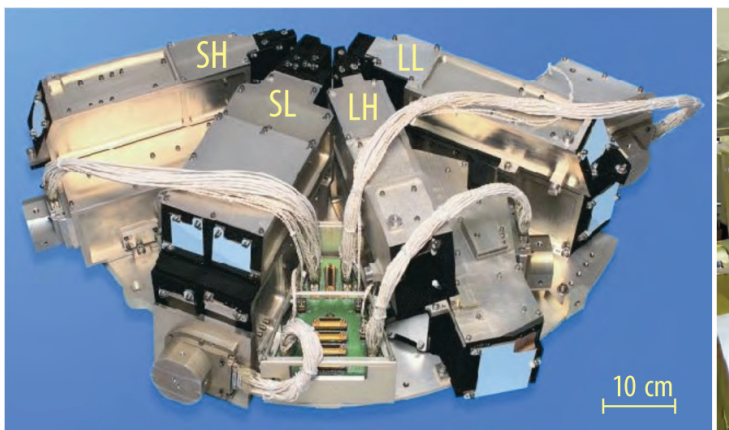

(a)

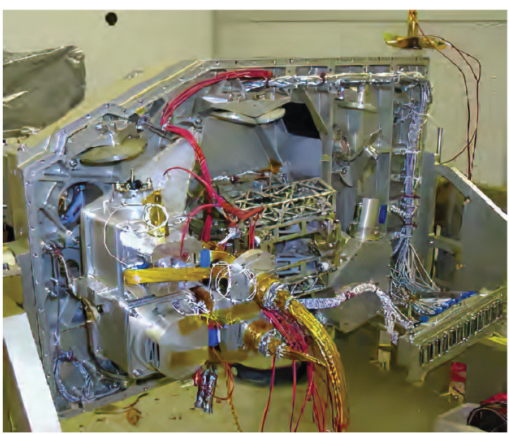

(b)

Fig. 12 Heritage for the basic approach of the OSS elements grating and FTS elements. The bolted-aluminum OSS grating spectrometer uses the same approach as the Spitzer infrared spectrograph from Ref. 76. (a) Its four wideband modules and (b) the Fourier-transform module path-folding approach and mechanism use the same approach as in the Herschel SPIRE instrument. $^{26}$

\section{Heritage and Risk Management}

The heritage of the OSS detector system, and its path to flight readiness, is outlined in Sec. 5 and detailed in the Origins Technology Development Plan. Here, we consider the heritage of the optical elements: the gratings, FTS, and etalon. The grating modules are straightforward bolted aluminum systems using diamond-machined mirrors and gratings. They have no moving parts. Similar approaches have been employed on the Spitzer infrared spectrograph [Fig. 12(a)] at even shorter (more demanding) wavelengths. FTSs have been used at these wavelengths in space in multiple instruments, including the Cosmic Background Experiment (COBE) and the SPIRE instrument on Herschel [Fig. 12(b)]. SPIRE's FTS also used multiple sets of rooftop mirrors on the moving stages to provide pathlength amplification.

Scanning Fabry-Perot etalons have been used successfully on European space agency's Infrared Space Observatory (ISO) mission; the short-wavelength spectrometer (SWS) and the long-wavelength spectrometer on ISO had scanning etalons [Fig. 13(a)] that were operated in front of the diffraction gratings. These etalons had higher finesse $(F=$ cavity quality factor $Q \sim 200$ ) for ISO SWS than the OSS (baseline $F=70$ ). They also operated at shorter wavelengths. Both these differences created more stringent requirements on the mirror flatness and parallelism than will be levied by the OSS. OSS does require a larger clear aperture than ISO, but this is comparable to that used on the ground-based submillimeter instrument SPIFI. ${ }^{77-79}$

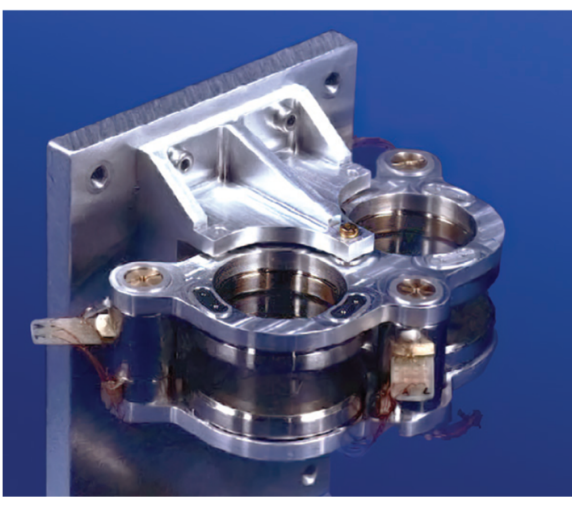

(a)

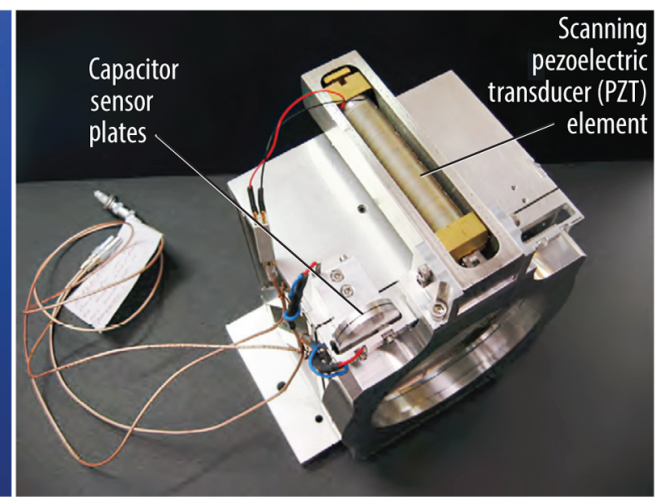

(b)

Fig. 13 Heritage of scanning etalons for far-IR astrophysics: (a) the dual etalon used in the ISO (credit Max Planck Institute for Extraterrestrial Physics, Garching). (b) An etalon developed at Cornell for use on SOFIA. ${ }^{29}$ It has demonstrated $R=100,000$ at $112 \mu \mathrm{m}$, using an order of 2000 and a cavity finesse of 50 . OSS will use a similar finesse, but larger cavity spacing. 
Finally, new high-resolution far-IR etalons are now being built for the HIRMES instrument on SOFIA [Fig. 13(b)], ${ }^{29}$ which are good proxies for the OSS etalon in terms of wavelength and cavity finesse.

In developing the concept, we have identified the most important risk areas for OSS and developed approaches to their mitigation.

1. The most important risks for OSS are in the detector system, in particular the sensitivity and yield at large array format, and susceptibility to effects on orbit such as cosmic rays. These aspects are addressed with a dedicated technology development plan ${ }^{63}$ (see Sec. 5) in advance of the flight program.

2. Stray light, magnetic susceptibility, and rf power are known potential problems with cryogenic superconducting detectors. The stray light risk is mitigated with the design; the grating spectrometer assemblies are light tight with only the slit as entrance points; they can be tested independently with radiative sources (loads) outside the slit to verify that no excess power is coupled to the detectors beyond what is expected in-band from the spectrometer optics. The magnetic susceptibility risk, while often a problem in ground-based and suborbital instruments and important to understand early in the design cycle, is also straightforward to assess and mitigate for OSS. The ambient magnetic field at L2 is much smaller than Earth's field, so the most important field sources are those on board the Origins spacecraft (e.g., the ADR magnets), but these will be shielded. Their effect can be bounded cleanly because field strength decays as $1 /$ distance $^{3}$. The susceptibility of the focal plane packages can be separately measured with ac-modulated Helmhotz coils in the lab; and the susceptibility can be shown to be 1 to 2 orders of magnitude lower that what would impact scientific performance. The rf power may be most challenging among these if bolometers are used. rf power can couple to bolometers directly, generating an analog of stray light, and the rf environment can depend on details of the wiring harness and other observatory-level aspects, which are hard to test prior to instrument integration. Here again the grating module boxes help provide a form of mitigation-they will be used as approximate Faraday cages sealed except for the slit entrance), with filtered electrical feedthroughs for all bias and readout lines. Additionally, the team will carry out early tests with flight-like cables, electronics, and other identified Origins rf sources (or suitable proxies), at the individual OSS grating module level in phase $\mathrm{C}$, prior to integration.

3. A final class of system-level performance risk is the failure of mechanisms. The impact of this risk is limited, since much of the science is done in the grating-only modes, which do not require any moving parts with the exception of the Origins steering mirror (FSM) for some modes. The likelihood of this risk materializing is to be mitigated using highheritage approaches, as well as early design, prototyping, and qualification including cryogenic testing. The FTS insertion carriage will be designed to fail in the "in" position, so that the interferometer can be used even if the mechanism fails. (The base grating mode is still operable in this condition because the slits are larger than the inteferometer pickoff mirror.) By the same principle, the etalon insertion mechanism is design to fail in the "out" position, to leave the FTS path clear.

\section{Summary}

Spectroscopy in the far-IR offers tremendous opportunity for answering fundamental questions in galaxy evolution and planet formation. In far-IR spectroscopy, a revolution in capability is available with an actively cooled telescope and large-format far-IR detectors. Origins and the OSS are designed to bring this to fruition. OSS is a suite of six long-slit $R=300$ grating modules, which combine to cover the full 25 to $588 \mu \mathrm{m}$ range simultaneously, operating with sensitivity approaching the photon background limit in each of a total of 60,000 pixels in the six arrays. The wideband grating modules are simple machined aluminum structures with no moving parts, the same approach as that used on the Spitzer IRS. OSS will be a powerful mapping instrument, providing full-band spectra of millions of galaxies in unbiased surveys. Additionally, to enable detailed studies of individual objects, an interferometer can be engaged to process light from a piece of the grating field of view with an FTS, which also covers the full OSS band and 
provides $R$ up to 43,000 at $112 \mu \mathrm{m}$. For kinematic studies in disks, an etalon is also provided. It can be inserted into the interferometer beam to provide 1 to $2 \mathrm{~km} \mathrm{~s}^{-1}$ resolution over the 100-to $200-\mu \mathrm{m}$ sub-band. Though large, the instrument is tractable. A detector maturation plan has been developed and all other technical aspects of OSS have heritage. Our point design which includes optical, mechanical, electrical, and thermal aspects closes with margin in the Origins accommodations.

\section{Acknowledgments}

We acknowledge the hard work of the GSFC Instrument Design Laboratory (IDL) in fleshing out the OSS concept and the Origins Science and Technology Definition Team (STDT) for help in developing science cases and defining instrument parameters. We also acknowledge the useful conversations with Willem Jellema and David Naylor regarding the Martin-Puplett interferometer. Finally, we thank two anonymous referees for their insightful comments, which improved the quality of the article. A portion of this research was carried out at the Jet Propulsion Laboratory of California Institute of Technology, under a contract with the National Aeronautics and Space Administration (Grant No. 80NM0018D0004). Government sponsorship also acknowledged.

\section{References}

1. G. J. Stacey, "Far-infrared spectroscopy diagnostics of the interstellar medium in galaxies," Astron. Soc. Pac. Conf. Ser. 41, 297 (1993).

2. J. W. Colbert et al., "ISO LWS spectroscopy of M82: a unified evolutionary model," Astrophys. J. 511, 721-729 (1999).

3. D. A. Dale et al., "The Spitzer infrared nearby galaxies survey: a high-resolution spectroscopy anthology," Astrophys. J. 693, 1821-1834 (2009).

4. P. Ogle et al., "Ultraluminous star-forming galaxies and extremely luminous warm molecular hydrogen emission at $z=2.16$ in the PKS 1138-26 radio galaxy protocluster," Astrophys. J. 751, 13 (2012).

5. P. Ogle et al., "Jet-powered molecular hydrogen emission from radio galaxies," Astrophys. J. 724, 1193-1217 (2010)

6. J. D. T. Smith et al., "The mid-infrared spectrum of star-forming galaxies: global properties of polycyclic aromatic hydrocarbon emission," Astrophys. J. 656, 770-791 (2007).

7. P. R. Roelfsema et al., "SPICA-a large cryogenic infrared space telescope: unveiling the obscured universe," Pub. Astron. Soc. Aust. 35, e030 (2018).

8. W. Jellema et al., "SAFARI: instrument design of the far-infrared imaging spectrometer for SPICA," Proc. SPIE 10563, 105631K (2017).

9. J. Glenn et al., "The Galaxy evolution probe: a concept for a mid and far-Infrared Space Observatory," Proc. SPIE 10698, 106980L (2018).

10. A. Pope et al., "Origins Space Telescope: 3D infrared surveys of star formation and black hole growth in galaxies over cosmic time," in Am. Astron. Soc. Meeting Abstracts, Vol. 231, p. 355.50 (2018).

11. E. Visbal and A. Loeb, "Measuring the 3D clustering of undetected galaxies through cross correlation of their cumulative flux fluctuations from multiple spectral lines," J. Cosmol. Astropart. Phys. 2010, 16 (2010).

12. E. Visbal, H. Trac, and A. Loeb, "Demonstrating the feasibility of line intensity mapping using mock data of galaxy clustering from simulations," J. Cosmol. Astropart. Phys. 2011, 10 (2011).

13. Y. Gong et al., "Probing reionization with intensity mapping of molecular and fine-structure lines," Astrophys. J. Lett. 728, L46 (2011).

14. Y. Gong et al., "Intensity mapping of the [C II] fine structure line during the epoch of reionization," Astrophys. J. 745, 49 (2012).

15. Y. Gong, A. Cooray, and M. G. Santos, "Probing the pre-reionization epoch with molecular hydrogen intensity mapping," Astrophys. J. 768, 130 (2013). 
Bradford et al.: Origins Survey Spectrometer: revealing the hearts of distant galaxies...

16. B. D. Uzgil et al., "Measuring galaxy clustering and the evolution of [C II] mean intensity with far-IR line intensity mapping during $0.5<z<1.5$," Astrophys. J. 793, 116 (2014).

17. P. Serra, O. Doré, and G. Lagache, "Dissecting the high-z interstellar medium through intensity mapping cross-correlations," Astrophys. J. 833, 153 (2016).

18. L. Armus et al., "Measuring galactic feedback with the Origins Space Telescope," in Am. Astron. Soc. Meeting Abstracts, Vol. 231, p. 355.05 (2018).

19. S. Hanany et al., "Millimeter-wave achromatic half-wave plate," Appl. Opt. 44, 4666-4670 (2005).

20. J. Zhang et al., "Polypropylene embedded metal mesh broadband achromatic half-wave plate for millimeter wavelengths," Appl. Opt. 50, 3750-3757 (2011).

21. G. Pisano et al., "Achromatic half-wave plate for submillimeter instruments in cosmic microwave background astronomy: experimental characterization," Appl. Opt. 45, 6982-9 (2006).

22. K. Komatsu et al., "Demonstration of the broadband half-wave plate using the nine-layer sapphire for the cosmic microwave background polarization experiment," J. Astron. Telesc. Instrum. Syst. 5(4), 1-14 (2019).

23. C. Gandhi, P. R. Babu, and K. Senthilnathan, "Designing a broadband terahertz half-wave plate using an anisotropic metasurface," J. Infrared Millimeter Terahertz Waves 40(5), 500-515 (2019).

24. R.-H. Fan et al., "Freely tunable broadband polarization rotator for terahertz waves," Adv. Mater. 27(7), 1201-1206 (2015).

25. C. Sabah and H. Roskos, "Design of a terahertz polarization rotator based on a periodic sequence of chiral-metamaterial and dielectric slabs," Prog. Electromagn. Res. 124, 301 (2012).

26. M. J. Griffin et al., "The Herschel-SPIRE instrument and its in-flight performance," Astron. Astrophys. 518, L3 (2010).

27. D. H. Martin and E. Puplett, "Polarised interferometric spectrometry for the millimeter and submillimeter spectrum," Infrared Phys. 10, 105-109 (1970).

28. D. K. Lambert and P. L. Richards, "Martin-Puplett interferometer: an analysis," Appl. Opt. 17, 1595-1602 (1978).

29. G. Douthit et al., "Development of the Fabry-Perot interferometers for the HIRMES spectrometer on SOFIA," Proc. SPIE 10708, 107081P (2018).

30. N. F. Cothard et al., "Optimizing the efficiency of Fabry-Perot interferometers with siliconsubstrate mirrors," Proc. SPIE 10706, 107065B (2018).

31. P. Nagler, J. Sadleir, and E. Wollack, "Transition-edge sensor detectors for the Origins Space Telescope," J. Astron. Telesc. Instrum. Syst. 7(1), 011005 (2021).

32. W. S. Holland et al., "SCUBA-2: the 10000 pixel bolometer camera on the James Clerk Maxwell Telescope," Mon. Not. R. Astron. Soc. 430, 2513-2533 (2013).

33. Z. Staniszewski et al., "The Keck array: a multi camera CMB polarimeter at the South Pole," J. Low Temp. Phys. 167, 827-833 (2012).

34. J. G. Staguhn et al., "The gismo two-millimeter deep field in GOODS-N," Astrophys. J. 790, 77 (2014).

35. C. Ferkinhoff et al., "The second-generation z (Redshift) and early universe spectrometer. I. First-light observation of a highly lensed local-Ulirg analog at high-z," Astrophys. J. 780, 142 (2014).

36. R. Gualtieri et al., "SPIDER: CMB polarimetry from the edge of space," J. Low Temp. Phys. 193, 1112-1121 (2018).

37. A. D. Beyer et al., "Ultra-sensitive transition-edge sensors for the background limited infrared/sub-mm spectrograph (BLISS)," J. Low Temp. Phys. 167, 182-187 (2012).

38. P. Khosropanah et al., "Ultra-low noise TES bolometer arrays for SAFARI instrument on SPICA," Proc. SPIE 9914, 99140B (2016).

39. J. A. Chervenak et al., "Superconducting multiplexer for arrays of transition edge sensors," Appl. Phys. Lett. 74(26), 4043-4045 (1999).

40. K. Irwin et al., "Microwave squid multiplexers for low-temperature detectors," Nucl. Instrum. Methods Phys. Res. Sec. A 559(2), 802-804 (2006). 
Bradford et al.: Origins Survey Spectrometer: revealing the hearts of distant galaxies...

41. S. R. Dicker et al., "Mustang 2: a large focal plane array for the $100 \mathrm{~m}$ green bank telescope,' J. Low Temp. Phys. 176(5), 808-814 (2014).

42. M. A. Dobbs et al., "Frequency multiplexed superconducting quantum interference device readout of large bolometer arrays for cosmic microwave background measurements," Rev. Sci. Instrum. 83(7), 073113 (2012).

43. J. van der Kuur et al., "Implementation of frequency domain multiplexing in imaging arrays of microcalorimeters," Nucl. Instrum. Methods Phys. Res. Sec. A 520(1), 551-554 (2004).

44. J. van der Kuur et al., "Small-signal behavior of a TES under ac bias," IEEE Trans. Appl. Supercond. 21, 281-284 (2011).

45. S. Hailey-Dunsheath et al., "Kinetic inductance detectors for the origins space telescope," $J$. Astron. Telesc. Instrum. Syst. 7(1) (2021).

46. P. K. Day et al., "A broadband superconducting detector suitable for use in large arrays," Nature 425, 817-821 (2003).

47. P. R. Maloney et al., "MUSIC for sub/millimeter astrophysics," Proc. SPIE 7741, 77410F (2010).

48. B. A. Mazin et al., "ARCHONS: a highly multiplexed superconducting optical to near-IR camera," Proc. SPIE 7735, 773518 (2010).

49. A. Monfardini et al., "A dual-band millimeter-wave kinetic inductance camera for the IRAM 30 m telescope," Astrophys. J. Suppl. Ser. 194, 24 (2011).

50. L. J. Swenson et al., "MAKO: a pathfinder instrument for on-sky demonstration of low-cost 350 micron imaging arrays," Proc. SPIE 8452, 84520P (2012).

51. R. Adam et al., "The NIKA2 large-field-of-view millimetre continuum camera for the $30 \mathrm{~m}$ IRAM telescope," Astron. Astrophys. 609, A115 (2018).

52. J. E. Austermann et al., "Millimeter-wave polarimeters using kinetic inductance detectors for TolTEC and beyond," J. Low Temp. Phys. 193, 120-127 (2018).

53. N. Galitzki et al., "Instrumental performance and results from testing of the BLAST-TNG receiver, submillimeter optics, and MKID detector arrays," Proc. SPIE 9914, 99140J (2016).

54. J. J. A. Baselmans et al., "A kilo-pixel imaging system for future space based far-infrared observatories using microwave kinetic inductance detectors," Astron. Astrophys. 601, A89 (2017).

55. S. Hailey-Dunsheath et al., "Development of aluminum LEKIDs for balloon-borne far-IR spectroscopy," J. Low Temp. Phys. 193, 968-975 (2018).

56. P. M. Echternach, A. D. Beyer, and C. M. Bradford, "Large array of low-frequency readout quantum capacitance detectors," J. Astron. Telesc. Instrum. Syst. 7(1), 011003 (2021).

57. P. M. Echternach et al., "Photon shot noise limited detection of terahertz radiation using a quantum capacitance detector," Appl. Phys. Lett. 103, 053510 (2013).

58. P. M. Echternach et al., "Single photon detection of $1.5 \mathrm{THz}$ radiation with the quantum capacitance detector," Nat. Astron. 2, 90-97 (2018).

59. J. A. B. Mates et al., "Simultaneous readout of 128 x-ray and gamma-ray transition-edge microcalorimeters using microwave squid multiplexing," Appl. Phys. Lett. 111(6), 062601 (2017).

60. B. Dober et al., "Microwave squid multiplexer demonstration for cosmic microwave background imagers," Appl. Phys. Lett. 111(24), 243510 (2017).

61. B. Farley et al., "A programmable RFSoC in 16nm FinFET technology for wideband communications," in IEEE Asian Solid-State Circuits Conf., pp. 1-4 (2017).

62. F. Hsiao et al., "A 2.2 Gs/s $188 \mathrm{mw}$ spectrometer processor in 65nm CMOS for supporting low-power THz planetary instruments," in IEEE Custom Integrated Circuits Conf., pp. 1-3 (2015).

63. Origins study team, "Origins volume 2: technology development plan," https://asd.gsfc.nasa .gov/firs/docs/Origins Volume2TechDevelopmentPlanREDACTED.pdf (August 2019).

64. C. M. Bradford et al., "The warm molecular gas around the Cloverleaf Quasar," Astrophys. J. 705, 112-122 (2009).

65. G. J. Stacey et al., "ZEUS: the redshift (z) and early Universe spectrometer," Proc. SPIE 375, 52 (2007).

66. P. J. Shirron et al., A Multi-Stage Continuous-Duty Adiabatic Demagnetization Refrigerator, pp. 1629-1638, Springer US, Boston, MA (2000). 
Bradford et al.: Origins Survey Spectrometer: revealing the hearts of distant galaxies...

67. J. Tuttle et al., "Development of a space-flight ADR providing continuous cooling at $50 \mathrm{mK}$ with heat rejection at 10 k," IOP Conf. Ser. Mater. Sci. Eng. 278, 012009 (2017).

68. P. J. Shirron et al., "Operating modes and cooling capabilities of the 3 -stage ADR developed for the soft-x-ray spectrometer instrument on ASTRO-H," Cryogenics 74, 2-9 (2016).

69. B. Swinyard et al., "FIRST-SPIRE spectrometer a novel imaging FTS for the sub-millimeter," Proc. SPIE 4013, 196-207 (2000).

70. K. Dohlen et al., "Optical design of the SPIRE instrument for FIRST," Proc. SPIE 4013, 119-128 (2000).

71. D.W. Walser and E. Donald, Jennings of Goddard Space Flight Center, "Double-parallelogram carriage for spectrometer mirrors," NASA Tech Briefs, https://www.techbriefs.com/ component/content/article/tb/supplements/mctb/briefs/29509 (1 August 2001).

72. Kaman Precision Products, "DIT-5200," Kaman Product Information, https://www .kamansensors.com/product/dit-5200/ (2021).

73. Mad City Labs, "Single-axis piezo nanopositioners," MCL product information, http://www .madcitylabs.com/nanopositioners.html (2021).

74. J.-M. Lamarre et al., "Planck high-frequency instrument," Proc. SPIE 4850, 730-739 (2003).

75. Planck HFI Core Team et al., "Planck early results. VI. The high frequency instrument data processing," Astron. Astrophys. 536, A6 (2011).

76. J. R. Houck et al., "The infrared spectrograph on the Spitzer Space Telescope," Proc. SPIE 5487, 62-76 (2004).

77. C. M. Bradford et al., "CO $(J=7-6)$ observations of NGC 253: cosmic-ray-heated warm molecular gas," Astrophys. J. 586, 891-901 (2003).

78. C. M. Bradford et al., "WaFIRS: a waveguide far-IR spectrometer: enabling spectroscopy of high-z galaxies in the far-IR and submillimeter," Proc. SPIE 4850, 1137-1148 (2003).

79. T. E. Oberst et al., "A $205 \mu \mathrm{m}$ [N II] map of the Carina Nebula," Astrophys. J. 739, 100 (2011).

Charles Matt Bradford received his doctorate degree in astronomy and instrumentation from Cornell in 2001. He held a Millikan postdoctoral fellowship at Caltech from 2001 to 2003 and has been on the science staff at JPL since that time. He enjoys developing and fielding new submillimeter- and millimeter-wave instrumentation on mountain-top sites, using the datasets to study interstellar medium conditions in galaxies near and far. Current projects include a balloonborne far-IR spectrometer, on-chip mm-wave spectrometer demonstration, and a mm-wave line intensity mapping instrument. He is also active in developing concepts and the necessary detector systems for cryogenic space far-IR astrophysics missions.

Damon Bradley is a head of the Instrument Electronics Development Branch and a founder of the Digital Signal Processing Technology Group at NASA Goddard Space Flight Center, where he supervises the research and development work of more than 70 engineers and 30 projects. $\mathrm{He}$ is also a coinvestigator of three different spaceflight spectrometer systems and serves as an adjunct professor in the Electrical and Computer Engineering Department at University of Maryland, Baltimore County.

David Leisawitz is a NASA study scientist for the Origins Space Telescope and is most interested scientifically in the development of habitable conditions during planet formation. He was a principal investigator (PI) on the Space Infrared Interferometric Telescope mission concept study and served as NASA Goddard study lead for the Submillimeter Probe of the Evolution of Cosmic Structure. He was mission scientist for the Wide-field Infrared Survey Explorer and Deputy Project Scientist for the Cosmic Background Explorer.

Michael Dipirro joined NASA Goddard in 1980, after receiving a $\mathrm{PhD}$ in low-temperature physics from the State University of NY at Buffalo, and a 1-year NRC postdoctoral fellowship at NIST. He has worked on a number of astrophysics missions over the last 40 years including COBE, ASTRO-E, -E2, and -H, XRISM, Spitzer, WIRE, WISE, and JWST. Between COBE and ASTRO-E, he was the PI on the Superfluid Helium On-Orbit Transfer Flight Demonstration, and Co-I on a Cross Enterprise Technology Development Program to develop a new type of adiabatic 
demagnetization refrigerator. He is currently the technical lead and chief technologist for the Origins Space Telescope study for the 2020 Astrophysics Decadal Survey.

Alexandra Pope PhD University of British Columbia is an associate professor of Astronomy at the University of Massachusetts Amherst. She is an observational astronomer who specializes in infrared, (sub)millimeter and radio observations of dusty galaxies at high redshift.

Margaret Meixner received her bachelor's degree in electrical engineering and math in 1987 from the University of Maryland, College Park, Maryland, USA, and her PhD in astronomy in 1993 from the University of California, Berkeley, California, USA. She is a director of the SOFIA Science Mission Operations Center. Her research includes infrared instrumentation and dust evolution in galaxies. She is a member of the JWST/MIRI Science Team and a community cochair of NASA's STDT for the Origins Space Telescope mission concept. Formerly, she was a JWST project scientist and distinguished astronomer at STScI. She is a fellow of the AAAS.

Biographies of the other authors are not available. 